\title{
Detailed balance condition and effective free energy in the primitive chain network model.
}

\section{$\operatorname{AUTHOR}(S)$ :}

Uneyama, Takashi; Masubuchi, Yuichi

\section{CITATION:}

Uneyama, Takashi ...[et al]. Detailed balance condition and effective free energy in the primitive chain network model.. The Journal of chemical physics 2011, 135(18): 184904.

\author{
ISSUE DATE: \\ 2011-11 \\ URL: \\ http://hdl.handle.net/2433/151709 \\ RIGHT: \\ (C) 2011 American Institute of Physics
}




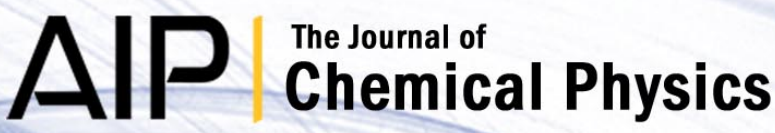

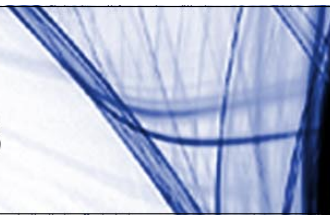

\section{Detailed balance condition and effective free energy in the primitive chain network model}

Takashi Uneyama and Yuichi Masubuchi

Citation: J. Chem. Phys. 135, 184904 (2011); doi: 10.1063/1.3658775

View online: http://dx.doi.org/10.1063/1.3658775

View Table of Contents: http://jcp.aip.org/resource/1/JCPSA6/v135/i18

Published by the American Institute of Physics.

\section{Related Articles}

Spatial distribution of intra-molecular water and polymeric components in polyelectrolyte dendrimers revealed by small angle scattering investigations

J. Chem. Phys. 135, 144903 (2011)

Spin coating of an evaporating polymer solution

Phys. Fluids 23, 102101 (2011)

Thermoreversible gelation of isotactic-rich poly( $\mathrm{N}$-isopropylacrylamide) in water

J. Chem. Phys. 135, 114903 (2011)

Comparison of Brownian dynamics algorithms with hydrodynamic interaction

J. Chem. Phys. 135, 084116 (2011)

Conformation of a flexible chain in explicit solvent: Exact solvation potentials for short Lennard-Jones chains

J. Chem. Phys. 135, 044903 (2011)

\section{Additional information on J. Chem. Phys.}

Journal Homepage: http://jcp.aip.org/

Journal Information: http://jcp.aip.org/about/about_the_journal

Top downloads: http://jcp.aip.org/features/most_downloaded

Information for Authors: http://jcp.aip.org/authors

\section{ADVERTISEMENT}

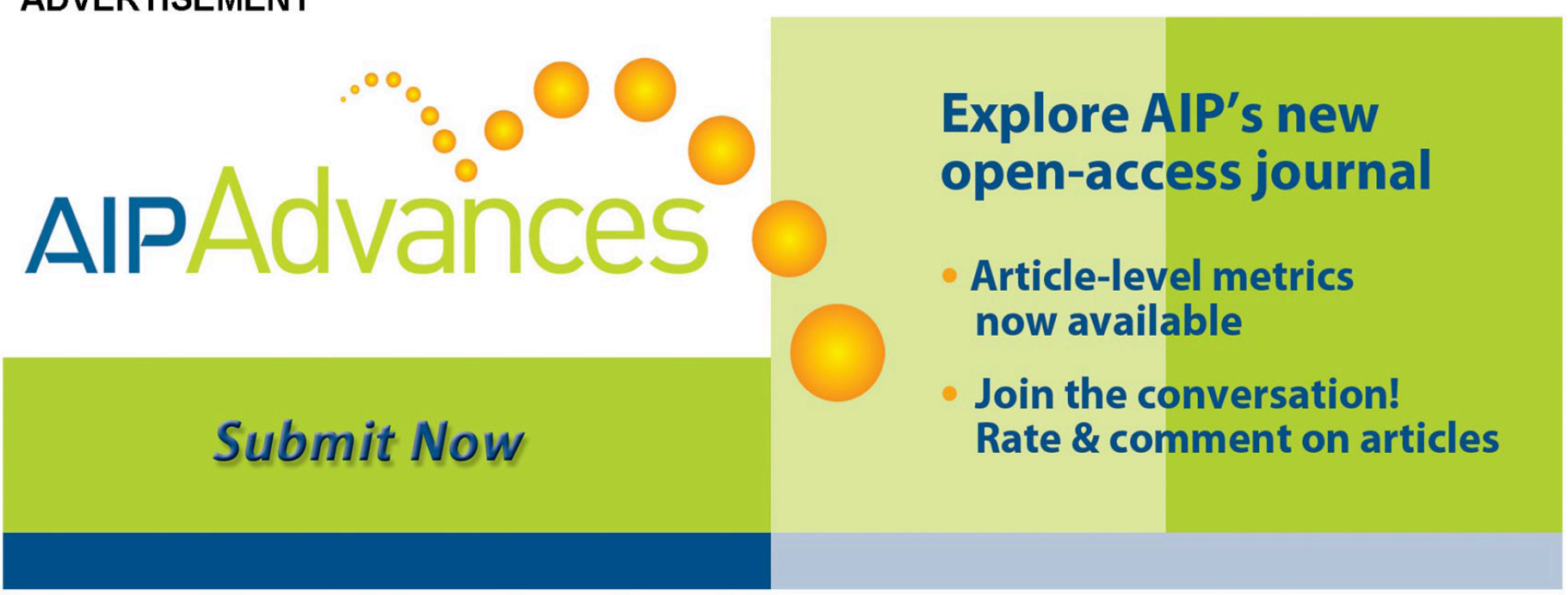




\title{
Detailed balance condition and effective free energy in the primitive chain network model
}

\author{
Takashi Uneyama ${ }^{\text {a) }}$ and Yuichi Masubuchi \\ JST-CREST, Institute for Chemical Research, Kyoto University, Gokasho, Uji, Kyoto 611-0011, Japan
}

(Received 10 July 2011; accepted 17 October 2011; published online 10 November 2011)

\begin{abstract}
We consider statistical mechanical properties of the primitive chain network (PCN) model for entangled polymers from its dynamic equations. We show that the dynamic equation for the segment number of the PCN model does not reduce to the standard Langevin equation which satisfies the detailed balance condition. We propose heuristic modifications for the PCN dynamic equation for the segment number, to make it reduce to the standard Langevin equation. We analyse some equilibrium statistical properties of the modified PCN model, by using the effective free energy obtained from the modified PCN dynamic equations. The PCN effective free energy can be interpreted as the sum of the ideal Gaussian chain free energy and the repulsive interaction energy between slip-links. By using the single chain approximation, we calculate several distribution functions of the PCN model. The obtained distribution functions are qualitatively different from ones for the simple sliplink model without any direct interactions between slip-links. (C) 2011 American Institute of Physics. [doi:10.1063/1.3658775]
\end{abstract}

\section{INTRODUCTION}

The primitive chain network (PCN) model ${ }^{1,2}$ is a sliplink type mesoscopic coarse-grained model for entangled polymeric systems. ${ }^{3}$ To simulate rheological properties efficiently, the PCN model represents entangled polymers as a network like structure of which topology dynamically changes. Simulations based on the PCN model can reproduce rheological properties of entangled polymers well with relatively small computational costs, and it has been applied to various systems including branched polymers ${ }^{4,5}$ or bidisperse polymers. ${ }^{6}$ Although the PCN model achieved success in predicting various rheological behaviours of entangled polymers, its statistical properties are still not fully understood.

In recent years, various primitive path analysis methods $^{7-9}$ have been developed to extract statistical properties of network structures in entangled polymers quantitatively from atomistic or coarse-grained molecular models (such as the molecular dynamics simulations). It is an interesting question whether the extracted statistical data agree with the ones obtained by the PCN model, to discuss consistency or relation between the models. Quite recently, the network statistics of the PCN model were systematically examined. ${ }^{10}$ The statistical properties of the PCN model are shown to be qualitatively in agreement with the primitive path analysis data. However, the analysis is conducted only for simulation results, and from the theoretical view point, how the network statistics is determined in the PCN model is still not clear. The PCN model is a dynamical model which is constructed in a rather phenomenological way. This makes theoretical analysis for the equilibrium statistics of the PCN model difficult. Moreover, the thermodynamic validity of

a) Author to whom correspondence should be addressed. Electronic mail: uneyama@scl.kyoto-u.ac.jp. the PCN model is not guaranteed from the view point of the statistical mechanics.

To achieve the thermal equilibrium state, the detailed balance condition is required to be satisfied. If the detailed balance condition is satisfied for a model, we can utilize the standard statistical mechanics to analyze the model. Namely, the equilibrium probability distribution is given by the Boltzmann type equilibrium distribution with the thermodynamic potential. In the vicinity of the equilibrium state, dynamical behaviours such as the relaxation or linear response properties can be related to the correlation functions of fluctuations in equilibrium. ${ }^{11-13}$ The detailed balance condition is a strong condition, and thus, it is not always satisfied in a phenomenologically constructed dynamical model. Therefore, it is desired to examine whether the PCN model satisfies the detailed balance condition or not. So far, most of the previous works for the PCN model focused on dynamical properties such as rheological properties. As far as the authors know, the analysis of the PCN model from the view point of statistical mechanics has never been shown explicitly in the literatures. (It would be fair to mention that several researchers have noticed that the PCN model or some other slip-link models do not satisfy the detailed balance condition, although it has not been stressed in published literatures. For some slip-link models, the statistical mechanical analysis or the modeling consistent with the detailed balance condition have been done. ${ }^{14,15}$ )

In this work, first we attempt to interpret the dynamic equations of the PCN model as the Langevin equations. We show that the PCN dynamic equations do not satisfy the detailed balance condition, and therefore, the PCN model does not have the thermal equilibrium state. Then, we propose possible modifications to recover the detailed balance condition, in a heuristic way. Even if we modify the PCN model, the resulting thermodynamic potential (effective free energy) of the PCN model is not identical to the free energy of the 
slip-link model without any direct interactions. In the PCN model, there is the effective repulsive interaction between neighboring slip-links on a polymer chain. Based on the obtained effective free energy and the single chain approximation, finally we calculate several equilibrium probability distribution functions analytically. We compare analytical results with other theories as well as PCN simulation data.

\section{STATISTICAL MECHANICAL INTERPRETATION OF PCN DYNAMIC EQUATIONS}

\section{A. PCN dynamic equations}

In the PCN model, ${ }^{1,2}$ entangled polymers are represented as a network structure which consists of nodes (slip-linked points and chain ends) and bonds which connect nodes. For simplicity, we limit ourselves to monodisperse linear polymer systems with the polymerization index (number of segments) being $N$. The state of the system is described by the set of coarse-grained variables: positions of slip-linked nodes and end nodes, numbers of segments between two neighboring nodes, and the connectivity information. For convenience, we express the index of the $k$ th node in the $i$ th chain as $(i, k)$. We express the position of the $(i, k)$ node as $\boldsymbol{R}_{i, k}$, and the number of segments between the $(i, k-1)$ and $(i, k)$ nodes as $N_{i, k}$. We also express the number of subchains in the $i$ th chain as $Z_{i}$. The 0 th and $Z_{i}$ th nodes in the $i$ th chain represent chain ends, whereas other nodes represent slip-linked nodes. A slip-linked node is assumed to be spatially coupled to the partner node. We express this by introducing a connectivity map for the node index, $C$ (the $(i, k)$ node is coupled to the $C(i, k)$ node). Two nodes connected by slip-links, $(i, k)$ and $C(i, k)$, share the same node position

$$
\boldsymbol{R}_{i, k}=\boldsymbol{R}_{C(i, k)} \quad \text { (for all slip-linked nodes). }
$$

The state of the system can be completely described by $\left\{\boldsymbol{R}_{i, k}\right\}$, $\left\{N_{i, k}\right\},\left\{Z_{i}\right\}$, and $C$. (The information of $C$ is not important in the following arguments, and thus, we do not describe it explicitly.)

In absence of external flow (deformation) field, the PCN dynamic equations $^{2}$ are described as follows: (Although there are several different versions of the PCN model, in this work we employ the version described in Ref. 2)

$$
\zeta \frac{d \boldsymbol{R}_{i, k}(t)}{d t}=\frac{3 k_{B} T}{b^{2}} \sum_{j, l}^{\prime} \frac{\boldsymbol{R}_{j, l}-\boldsymbol{R}_{i, k}}{N_{j, l}}+\sqrt{2 k_{B} T \zeta} \boldsymbol{w}_{i, k}^{(\boldsymbol{R})}(t),
$$

$$
\frac{d N_{i, k}(t)}{d t}= \begin{cases}-J_{i, 1}(t) & (k=1), \\ -J_{i, k}(t)+J_{i, k-1}(t) & \left(2 \leq k \leq Z_{i}-1\right) \\ J_{i, Z_{i}-1}(t) & \left(k=Z_{i}\right),\end{cases}
$$

$$
\begin{aligned}
\frac{\zeta}{2 \rho_{i, k}} J_{i, k}(t) \equiv & \frac{3 k_{B} T}{b^{2}}\left[\frac{\left|\boldsymbol{R}_{i, k+1}-\boldsymbol{R}_{i, k}\right|}{N_{i, k+1}}-\frac{\left|\boldsymbol{R}_{i, k}-\boldsymbol{R}_{i, k-1}\right|}{N_{i, k}}\right] \\
& +\sqrt{k_{B} T \zeta} w_{i, k}^{(N)}(t)
\end{aligned}
$$

where $\zeta$ is the friction coefficient of a node, $k_{B}$ is the Boltzmann constant, $T$ is the temperature, $b$ is the segment size, and $J_{i, k}(t)$ is the flux of the segment number on the $(i, k)$ node. The summation in the right-hand side of Eq. (2) is taken for all nodes connected to the target (topological neighbor nodes). The topological neighbor nodes are $(i, k \pm 1)$ and $\left(i^{\prime}\right.$, $\left.k^{\prime} \pm 1\right)\left(\right.$ with $\left.\left(i^{\prime}, k^{\prime}\right)=C(i, k)\right)$ for a slip-linked node, and $(i, k$ $+1)$ or $(i, k-1)$ for an end node. $\rho_{i, k}$ is the segment density along the polymer chain on the $(i, k)$ node and is defined as the arithmetic average of local densities in two neighboring bonds

$$
\rho_{i, k}\left(\left\{\boldsymbol{R}_{i, k}\right\},\left\{N_{i, k}\right\}\right) \equiv \frac{1}{2}\left[\frac{N_{i, k}}{\left|\boldsymbol{R}_{i, k}-\boldsymbol{R}_{i, k-1}\right|}+\frac{N_{i, k+1}}{\left|\boldsymbol{R}_{i, k+1}-\boldsymbol{R}_{i, k}\right|}\right] .
$$

$\boldsymbol{w}_{i, k}^{(\boldsymbol{R})}(t)$ and $w_{i, k}^{(N)}(t)$ are Gaussian white noises which satisfy the following relations:

$$
\begin{gathered}
\left\langle\boldsymbol{w}_{i, k}^{(\boldsymbol{R})}(t)\right\rangle=0, \quad\left\langle\boldsymbol{w}_{i, k}^{(\boldsymbol{R})}(t) \boldsymbol{w}_{j, l}^{(\boldsymbol{R})}\left(t^{\prime}\right)\right\rangle=\delta_{i j} \delta_{k l} \delta\left(t-t^{\prime}\right) \mathbf{1}, \\
\left\langle w_{i, k}^{(N)}(t)\right\rangle=0, \quad\left\langle w_{i, k}^{(N)}(t) w_{j, l}^{(N)}\left(t^{\prime}\right)\right\rangle=\delta_{i j} \delta_{k l} \delta\left(t-t^{\prime}\right), \\
\left\langle\boldsymbol{w}_{i, k}^{(\boldsymbol{R})}(t) w_{j, l}^{(N)}\left(t^{\prime}\right)\right\rangle=0,
\end{gathered}
$$

where $\langle\ldots\rangle$ means the statistical average and $\mathbf{1}$ is the unit tensor.

We note that the (osmotic) repulsive force terms ${ }^{1}$ are dropped in the dynamic equations (2)-(4). The repulsive force is required only to cancel the artificial attractive interaction in multi chain slip-link systems, ${ }^{16}$ and its contribution is not essential in the analysis in this work. (Actually, it is empirically known that as long as the repulsion is sufficiently strong to avoid aggregation, the PCN model shows almost the same statistics.)

To specify the PCN dynamics completely, we also need the network reconstruction rules. Whether the network is reconstructed or not is determined by the number of segments in chain end bonds. If the number of segments in a chain end bond becomes larger than a certain criterion, a new pair of slip-linked nodes are constructed. On the other hand, if the number of the segments in an chain end bond becomes smaller than another criterion, the slip-link attached to that bond is destructed. The criteria are given as $3 N_{0} / 2$ and $N_{0} / 2$, with $N_{0}$ being the average number of segments in a bond. Although the network reconstruction rules affect the statistics rather strongly, ${ }^{10}$ how they affect the statistics is not so clear. (It is reported that other reconstruction rules ${ }^{10,17}$ can improve several statistical properties.) In this work, we do not consider about the reconstruction process and concentrate on the dynamic equations for $\left\{\boldsymbol{R}_{i, k}\right\}$ and $\left\{N_{i, k}\right\}$ (Eqs. (2)-(4)).

\section{B. Detailed balance condition in the PCN model}

Because the PCN model is designed as a dynamical model, its static properties are not clear from its dynamic equations (2)-(4). Moreover, the existence of the thermodynamic potential (the free energy) is generally not guaranteed for such a phenomenological dynamical model. If 
Eqs. (2)-(4) satisfy the detailed balance condition, the forces are expressed as variations of the free energy. (In the followings, we call such forms as the variational forms.) Then, we can construct the free energy from Eqs. (2)-(4). In this section, we attempt to interpret the PCN dynamic equations as Langevin equations ${ }^{18}$ which satisfy the detailed balance condition.

According to the standard nonequilibrium statistical physics, ${ }^{13,19,20}$ dynamics of the system in the vicinity of equilibrium can be described well by the Langevin equations. If we assume that there is no dynamic coupling between $\left\{\boldsymbol{R}_{i, k}\right\}$ and $\left\{N_{i, k}\right\}$, nor the memory effect, the detailed-balanced Langevin equations can formally be described as follows:

$$
\begin{aligned}
\frac{d \boldsymbol{R}_{i, k}(t)}{d t}= & -\sum_{j, l} \boldsymbol{L}_{i, k ; j, l}^{(\boldsymbol{R})} \cdot \frac{\partial \mathcal{F}_{\mathrm{eff}}\left(\left\{\boldsymbol{R}_{i, k}\right\},\left\{N_{i, k}\right\},\left\{Z_{i}\right\}\right)}{\partial \boldsymbol{R}_{j, l}} \\
& +k_{B} T \sum_{j, l} \frac{\partial}{\partial \boldsymbol{R}_{j, l}} \cdot \boldsymbol{L}_{i, k ; j, l}^{(\boldsymbol{R})}+\boldsymbol{\xi}_{i, k}^{(\boldsymbol{R})}(t), \\
\frac{d N_{i, k}(t)}{d t}= & -\sum_{j, l} L_{i, k ; j, l}^{(N)} \frac{\partial \mathcal{F}_{\mathrm{eff}}\left(\left\{\boldsymbol{R}_{i, k}\right\},\left\{N_{i, k}\right\},\left\{Z_{i}\right\}\right)}{\partial N_{j, l}} \\
& +k_{B} T \sum_{j, l} \frac{\partial L_{i, k ; j, l}^{(N)}}{\partial N_{j, l}}+\xi_{i, k}^{(N)}(t) .
\end{aligned}
$$

Here $\boldsymbol{L}_{i, k ; j, l}^{(\boldsymbol{R})}$ and $L_{i, k ; j, l}^{(N)}$ are the mobility matrices, which may depend on stochastic variables such as $\left\{\boldsymbol{R}_{i, k}\right\}$ or $\left\{N_{i, k}\right\}$. (From Onsager's reciprocal relation, these mobility matrices are symmetric.) $\mathcal{F}_{\text {eff }}\left(\left\{\boldsymbol{R}_{i, k}\right\},\left\{N_{i, k}\right\},\left\{Z_{i}\right\}\right)$ is the effective free energy of the system, and the (generalized) forces are expressed as the derivatives of $\mathcal{F}_{\text {eff }} \cdot \boldsymbol{\xi}_{i, k}^{(\boldsymbol{R})}(t)$ and $\xi_{i, k}^{(N)}(t)$ are the Gaussian random noises which satisfy the the fluctuation-dissipation relations of the second kind. They can be expressed as follows, by using Gaussian white noises $\boldsymbol{w}_{i, k}^{\boldsymbol{R}}(t)$ and $w_{i, k}^{(N)}(t)$ (their statistical moments are given by Eqs. (6)-(8)):

$$
\begin{gathered}
\boldsymbol{\xi}_{i, k}^{(\boldsymbol{R})}(t)=\sqrt{2 k_{B} T} \sum_{j, l} \boldsymbol{B}_{i, k ; j, l}^{(\boldsymbol{R})} \cdot \boldsymbol{w}_{j, l}^{(\boldsymbol{R})}(t), \\
\xi_{i, k}^{(N)}(t)=\sqrt{2 k_{B} T} \sum_{j, l} B_{i, k ; j, l}^{(N)} w_{j, l}^{(N)}(t), \\
\sum_{m, n} \boldsymbol{B}_{i, k ; m, n}^{(\boldsymbol{R})} \cdot\left[\boldsymbol{B}_{j, l ; m, n}^{(\boldsymbol{R})}\right]^{\mathrm{T}}=\boldsymbol{L}_{i, k ; j, l}^{(\boldsymbol{R})}, \\
\sum_{m, n} B_{i, k ; m, n}^{(N)} B_{j, l ; m, n}^{(N)}=L_{i, k ; j, l}^{(N)},
\end{gathered}
$$

where $\boldsymbol{B}^{\mathrm{T}}$ represents the transposed matrix of $\boldsymbol{B}$. In Eqs. (9) and (10), the stochastic terms are interpreted following the Ito calculus. ${ }^{18}$ The second terms in the right-hand sides of Eqs. (9) and (10) are spurious (noise-induced) drift terms, which are required to satisfy the detailed balance condition (for the Ito form Langevin equations). The dynamics described by Eqs. (9) and (10) is guaranteed to reach the equilibrium state, of which statistics is simply determined by the effective free energy $\mathcal{F}_{\text {eff }}$.

The PCN dynamic equation for $\left\{\boldsymbol{R}_{i, k}\right\}$ (Eq. (2)) seems to be similar to the Langevin equation (9). Actually, it is straightforward to show that Eq. (2) can be reduced to Eq. (9) by setting

$$
\begin{gathered}
\boldsymbol{L}_{i, k ; j, l}^{(\boldsymbol{R})}=\frac{1}{\zeta} \delta_{i j} \delta_{k l} \mathbf{1}, \\
\boldsymbol{\xi}_{i, k}^{(\boldsymbol{R})}=\sqrt{\frac{2 k_{B} T}{\zeta}} \boldsymbol{w}_{i, k}^{(\boldsymbol{R})}(t),
\end{gathered}
$$

$$
\begin{aligned}
\mathcal{F}_{\text {eff }}\left(\left\{\boldsymbol{R}_{i, k}\right\},\left\{N_{i, k}\right\},\left\{Z_{i}\right\}\right)= & \mathcal{F}_{\text {eff }, 0}\left(\left\{\boldsymbol{R}_{i, k}\right\},\left\{N_{i, k}\right\},\left\{Z_{i}\right\}\right) \\
& +\mathcal{F}_{\text {eff }, 1}\left(\left\{N_{i, k}\right\},\left\{Z_{i}\right\}\right),
\end{aligned}
$$

$\mathcal{F}_{\text {eff }, 0}\left(\left\{\boldsymbol{R}_{i, k}\right\},\left\{N_{i, k}\right\},\left\{Z_{i}\right\}\right) \equiv \frac{3 k_{B} T}{2 b^{2}} \sum_{i} \sum_{k=1}^{Z_{i}} \frac{\left(\boldsymbol{R}_{i, k}-\boldsymbol{R}_{i, k-1}\right)^{2}}{N_{i, k}}$.

Here $\mathcal{F}_{\text {eff }, 0}\left(\left\{\boldsymbol{R}_{i, k}\right\},\left\{N_{i, k}\right\},\left\{Z_{i}\right\}\right)$ is the free energy for ideal linear springs, and $\mathcal{F}_{\text {eff, } 1}\left(\left\{N_{i, k}\right\},\left\{Z_{i}\right\}\right)$ is the remaining contribution to the free energy which is independent of $\left\{\boldsymbol{R}_{i, k}\right\}$.

On the other hand, the PCN dynamic equation for $\left\{N_{i, k}\right\}$ (Eq. (3) together with Eq. (4)) is different from the standard Langevin equation (10). The segment number flux in the PCN dynamic equation (Eq. (4)) can be rewritten as follows:

$$
\begin{aligned}
J_{i, k}(t)= & M_{i, k} \frac{3 k_{B} T}{\rho_{i, k} b^{2}}\left[\frac{\left|\boldsymbol{R}_{i, k+1}-\boldsymbol{R}_{i, k}\right|}{N_{i, k+1}}-\frac{\left|\boldsymbol{R}_{i, k}-\boldsymbol{R}_{i, k-1}\right|}{N_{i, k}}\right] \\
& +\sqrt{2 k_{B} T M_{i, k}} w_{i, k}(t),
\end{aligned}
$$

where we defined $M_{i, k}$ as

$$
M_{i, k}\left(\left\{\boldsymbol{R}_{i, k}\right\},\left\{N_{i, k}\right\}\right) \equiv \frac{2 \rho_{i, k}^{2}\left(\left\{\boldsymbol{R}_{i, k}\right\},\left\{N_{i, k}\right\}\right)}{\zeta} .
$$

Substituting Eq. (17) into Eq. (3), we have the following expression for the dynamic equation: (For simplicity, we consider the case of $2 \leq k \leq Z_{i}-1$ in Eq. (3). The generalization to $k=1, Z_{i}$ is straightforward.)

$$
\begin{aligned}
\frac{d N_{i, k}(t)}{d t} & =-M_{i, k} \frac{3 k_{B} T}{\rho_{i, k} b^{2}}\left[\frac{\left|\boldsymbol{R}_{i, k+1}-\boldsymbol{R}_{i, k}\right|}{N_{i, k+1}}-\frac{\left|\boldsymbol{R}_{i, k}-\boldsymbol{R}_{i, k-1}\right|}{N_{i, k}}\right] \\
& +M_{i, k-1} \frac{3 k_{B} T}{\rho_{i, k-1} b^{2}}\left[\frac{\left|\boldsymbol{R}_{i, k}-\boldsymbol{R}_{i, k-1}\right|}{N_{i, k}}-\frac{\left|\boldsymbol{R}_{i, k-1}-\boldsymbol{R}_{i, k-2}\right|}{N_{i, k-1}}\right] \\
& -\sqrt{2 k_{B} T M_{i, k}} w_{i, k}(t)+\sqrt{2 k_{B} T M_{i, k-1}} w_{i, k-1}(t) .
\end{aligned}
$$

Comparing the stochastic terms in Eqs. (19) and (10), we find that the mobility matrix $L_{i, k ; j, l}^{(N)}$ can be expressed by using $M_{i, k}$ as

$$
L_{i, k ; j, l}^{(N)}= \begin{cases}M_{i, k}+M_{i, k-1} & (i=j, l=k), \\ -M_{i, k-1} & (i=j, l=k-1), \\ -M_{i, k} & (i=j, l=k+1), \\ 0 & \text { (otherwise) }\end{cases}
$$

We also find that the deterministic terms in Eq. (19) cannot be reduced to the variational form in Eq. (10). Namely, the PCN model does not satisfy the detailed balance condition, and thus, it does not have the thermal equilibrium state. The steady state in the PCN model (in absence of external flow 
field) corresponds to a nonequilibrium steady state, where the energy input and the energy dissipation is balanced. ${ }^{20}$

Nevertheless, PCN simulations by the original dynamic equations can reproduce some equilibrium properties of entangled polymers reasonably. ${ }^{10}$ This implies that, although the PCN model is not detailed-balanced and does not have the equilibrium state, it works as a good approximation of a statistical mechanically sound, detailed-balanced dynamical model. Keeping this in mind, we consider to remedy the PCN model to satisfy the detailed balance condition in a heuristic way. From Eqs. (19) and (10), the variational form for the force can be realized, if the following relation holds:

$$
\begin{aligned}
& \frac{3 k_{B} T}{\rho_{i, k} b^{2}}\left[\frac{\left|\boldsymbol{R}_{i, k}-\boldsymbol{R}_{i, k-1}\right|}{N_{i, k}}-\frac{\left|\boldsymbol{R}_{i, k+1}-\boldsymbol{R}_{i, k}\right|}{N_{i, k+1}}\right] \\
& =\left(\frac{\partial}{\partial N_{i, k+1}}-\frac{\partial}{\partial N_{i, k}}\right) \mathcal{F}_{\text {eff }}\left(\left\{\boldsymbol{R}_{i, k}\right\},\left\{N_{i, k}\right\},\left\{Z_{i}\right\}\right) .
\end{aligned}
$$

Clearly, Eq. (21) does not hold, if we use Eq. (5) as the definition of $\rho_{i, k}$. Conversely, if we do not use Eq. (5) as the definition of $\rho_{i, k}$, it becomes possible to satisfy Eq. (21). Besides, there is no special reason to employ the arithmetic average form for $\rho_{i, k}$. (Different models, such as a constant value model, were also proposed and utilized. ${ }^{1}$ ) Thus, here we define the segment density along the chain $\rho_{i, k}$ to recover the variational form

$\rho_{i, k}\left(\left\{\boldsymbol{R}_{i, k}\right\},\left\{N_{i, k}\right\}\right) \equiv 2\left[\frac{\left|\boldsymbol{R}_{i, k+1}-\boldsymbol{R}_{i, k}\right|}{N_{i, k+1}}+\frac{\left|\boldsymbol{R}_{i, k}-\boldsymbol{R}_{i, k-1}\right|}{N_{i, k}}\right]^{-1}$.

The right-hand side of Eq. (22) is the harmonic average of $N_{i, k+1} /\left|\boldsymbol{R}_{i, k+1}-\boldsymbol{R}_{i, k}\right|$ and $N_{i, k} /\left|\boldsymbol{R}_{i, k}-\boldsymbol{R}_{i, k-1}\right|$, whereas the right-hand side of Eq. (5) is the arithmetic average of them. By using the new definition (22), it is straightforward to show that the condition (21) is satisfied and $\mathcal{F}_{\text {eff, } 1}=0$.

Now the PCN dynamic equation for $\left\{N_{i, k}\right\}$ (Eq. (19)) can be rewritten as follows:

$$
\frac{d N_{i, k}(t)}{d t}=-\sum_{j, l} L_{i, k ; j, l}^{(N)} \frac{\partial \mathcal{F}_{\mathrm{eff}, 0}\left(\left\{\boldsymbol{R}_{i, k}\right\},\left\{N_{i, k}\right\},\left\{Z_{i}\right\}\right)}{\partial N_{j, l}}+\xi_{i, k}^{(N)}(t)
$$

with

$$
\xi_{i, k}^{(N)}(t)=-\sqrt{2 k_{B} T M_{i, k}} w_{i, k}^{(N)}(t)+\sqrt{2 k_{B} T M_{i, k-1}} w_{i, k-1}^{(N)}(t) .
$$

Although Eq. (23) is similar to Eq. (10), the spurious drift term (the second term in the right-hand side of Eq. (10)) is missing in Eq. (23). Therefore, in addition to employ a new definition of $\rho_{i, k}$ (Eq. (22)), we add the spurious drift term to the segment number flux equation (Eq. (4)). We modify the definition for the segment flux (Eq. (4)) as

$$
\begin{aligned}
\frac{\zeta}{2 \rho_{i, k}} J_{i, k}(t) \equiv & \frac{3 k_{B} T}{b^{2}}\left[\frac{\left|\boldsymbol{R}_{i, k+1}-\boldsymbol{R}_{i, k}\right|}{N_{i, k+1}}-\frac{\left|\boldsymbol{R}_{i, k}-\boldsymbol{R}_{i, k-1}\right|}{N_{i, k}}\right] \\
& +v_{i, k}^{(N)}+\sqrt{k_{B} T \zeta} w_{i, k}^{(N)}(t),
\end{aligned}
$$

where $v_{i, k}^{(N)}$ is the spurious drift velocity defined as

$$
\begin{aligned}
v_{i, k}^{(N)} & \equiv 2 k_{B} T\left(\frac{\partial}{\partial N_{i, k+1}}-\frac{\partial}{\partial N_{i, k}}\right) \rho_{i, k} \\
& =4 k_{B} T \frac{N_{i, k}^{2}\left|\boldsymbol{R}_{i, k+1}-\boldsymbol{R}_{i, k}\right|-N_{i, k+1}^{2}\left|\boldsymbol{R}_{i, k}-\boldsymbol{R}_{i, k-1}\right|}{\left(N_{i, k}\left|\boldsymbol{R}_{i, k+1}-\boldsymbol{R}_{i, k}\right|+N_{i, k+1}\left|\boldsymbol{R}_{i, k}-\boldsymbol{R}_{i, k-1}\right|\right)^{2}} .
\end{aligned}
$$

The modified PCN dynamic equation for $\left\{N_{i, k}\right\}$ (Eq. (3) together with Eqs. (22), (25), and (26)) reduces to Eq. (10). Thus, the modified PCN dynamic equations satisfy the detailed balance condition. It should be emphasized here that the noise term in Eq. (10) is the multiplicative noise because $L_{i, k ; j, l}^{(N)}$ depends on $\left\{N_{i, k}\right\}$ (through $M_{i, k}$ and $\rho_{i, k}$ ). Although the spurious drift term may not be intuitive, it naturally arises as a property of the multiplicative noise, ${ }^{13,21}$ and is required to satisfy the detailed balance condition.

Before we proceed to detailed analysis, here we shortly comment on the relation of our model to the GENERIC framework in the nonequilibrium thermodynamics. ${ }^{22,23}$ In our modified PCN dynamic equations, the thermodynamic forces are coupled to the symmetric mobility matrices. Such dynamic equations can straightforwardly be mapped onto the GENERIC framework. The mobility matrices and the effective free energy in our model correspond to the friction matrices and the entropy (with negative sign), respectively. They form the irreversible, dissipative bracket parts. The reversible, Poisson bracket parts do not exist in the modified PCN dynamic equations. Most of important properties in the nonequilibrium thermodynamics, such as the positivity of the entropy production, are automatically reproduced in the modified PCN dynamic equations. (Notice that, however, this does not mean that the modified PCN model is fully consistent with the GENERIC framework and the nonequilibrium thermodynamics. The repulsive interaction or the network reconstruction process may be inconsistent with them.)

\section{Effective free energy}

The modified PCN dynamic equations introduced in Sec. II B reduces to the detailed-balanced Langevin equation. The equilibrium state of the modified PCN model is determined by the effective free energy $\mathcal{F}_{\text {eff }}$. The effective free energy of the modified PCN model can simply be expressed as

$$
\begin{aligned}
\mathcal{F}_{\text {eff }}\left(\left\{\boldsymbol{R}_{i, k}\right\},\left\{N_{i, k}\right\},\left\{Z_{i}\right\}\right) & =\mathcal{F}_{\text {eff }, 0}\left(\left\{\boldsymbol{R}_{i, k}\right\},\left\{N_{i, k}\right\},\left\{Z_{i}\right\}\right) \\
& =\frac{3 k_{B} T}{2 b^{2}} \sum_{i} \sum_{k=1}^{Z_{i}} \frac{\left(\boldsymbol{R}_{i, k}-\boldsymbol{R}_{i, k-1}\right)^{2}}{N_{i, k}} .
\end{aligned}
$$

Notice that the effective free energy (27) is different from the free energy of the ideal Gaussian chains. The free energy of the Gaussian chains is expressed as

$$
\begin{aligned}
\mathcal{F}_{0}\left(\left\{\boldsymbol{R}_{i, k}\right\},\left\{N_{i, k}\right\},\left\{Z_{i}\right\}\right) \equiv & \frac{3 k_{B} T}{2 b^{2}} \sum_{i} \sum_{k=1}^{Z_{i}} \frac{\left(\boldsymbol{R}_{k}-\boldsymbol{R}_{k-1}\right)^{2}}{N_{k}} \\
& +\frac{3 k_{B} T}{2} \sum_{i} \sum_{k=1}^{Z_{i}} \ln N_{k} .
\end{aligned}
$$


Comparing Eqs. (27) and (28), we find that the logarithm term is missing in the PCN effective free energy.

We can rewrite the PCN effective free energy (Eq. (27)) as follows, by using the free energy of Gaussian chains (Eq. (28))

$$
\begin{aligned}
\mathcal{F}_{\text {eff }}\left(\left\{\boldsymbol{R}_{i, k}\right\},\left\{N_{i, k}\right\},\left\{Z_{i}\right\}\right)= & \mathcal{F}_{0}\left(\left\{\boldsymbol{R}_{i, k}\right\},\left\{N_{i, k}\right\},\left\{Z_{i}\right\}\right) \\
& +\sum_{i} \sum_{k=1}^{Z_{i}} \tilde{U}_{\text {slip-link }}\left(N_{k}\right), \\
\tilde{U}_{\text {slip-link }}(n) \equiv & -\frac{3 k_{B} T}{2} \ln n .
\end{aligned}
$$

Here $\tilde{U}_{\text {slip-link }}(n)$ represents the effective interaction between neighboring slip-links. (Strictly speaking, $\tilde{U}_{\text {slip-link }}$ also represents the effective interaction potential between a slip-link and a chain end. In the following, we assume that the interaction potentials between the slip-links and between a slip-link and a chain end are always the same.) We note that $\tilde{U}_{\text {slip-link }}(n)$ depends only on the number of segments between slip-links. This means that the effective interaction between slip-links is determined by the chemical distance, not by the spatial distance. Equation (30) is monotonically decreasing function of $n$, and thus, the interaction between slip-links is purely repulsive. In addition, the effective potential diverges at the limit of $n \rightarrow 0$, and thus, slip-links strongly repel each other at the short range (at the short chemical distance).

The second term in the right-hand side of Eq. (29) can be interpreted as the total repulsive interaction energy of sliplinks. In this sense, we can say that the PCN model is based on the Gaussian chain and repulsive slip-links. It is worth mentioning that such an effective repulsion between slip-links is already suggested by the CReTA primitive path extraction for Monte Carlo simulation data. ${ }^{9}$

\section{EQUILIBRIUM PROBABILITY DISTRIBUTIONS BY SINGLE CHAIN APPROXIMATION}

\section{A. Single chain approximation}

In Sec. II the effective free energy for the modified PCN model (Eq. (27)) is shown to be different from the free energy for the Gaussian chains (Eq. (28)) due to the repulsive interaction between slip-links. This means that several equilibrium statistical properties of the PCN model are qualitatively different from the ones of the Gaussian chains with slip-links without any direct interactions. However, unfortunately, we cannot calculate the equilibrium statistics of PCN analytically from the effective free energy. (It is practically impossible to calculate the statistical weight for strongly slip-linked multi chain systems.) In this section, we calculate the equilibrium statistical properties by using the mean field type single chain approximation. (We notice that it is not clear whether the single chain approximation employed in this work is really reasonable for the PCN model or not. We employ the simplest approximation to make the expressions simple and analytically tractable.)
Schieber ${ }^{24}$ proposed a single chain model with slip-links in which slip-links behave as a sort of grand canonical gas particles on a polymer chain. In his model, the number of slip-linked subchains is controlled by the effective chemical potential. There is no direct interaction between the slip-links, and the polymer chain obeys the Gaussian statistics. Namely, slip-links are placed on the target polymer chain and they do not directly interact with each other via a potential (this model corresponds to a sort of ideal model). In this work, we call his model as "the single chain non-interacting slip-link model" or simply as "the non-interacting slip-link model." (The expression "non-interacting" may sound somehow un-natural because the slip-links constrain the polymer chain and this constraint effect can be interpreted as a sort of interaction. Nonetheless, in this work we employ this expression because in the followings we consider another model in which sliplinks directly "interact" with each other.) The statistics of the single chain non-interacting slip-link model is briefly summarized in Appendix A.

Here, we follow Schieber's idea and consider the single chain version of the modified PCN model. We may call this as "the single chain repulsive slip-link model" or "the repulsive slip-link model" in the followings. This is because in our model, neighboring slip-links interact each other via the repulsive potential (Eq. (30)). In the single chain repulsive sliplink model, the state of the system is expressed by the node positions $\left\{\boldsymbol{R}_{k}\right\}$ ( $k$ represents the $k$ th segment on the chain), the numbers of segments $\left\{N_{k}\right\}$, and the number of subchains $Z$. (Under the mean field type single chain approximation, the connectivity map is not required.)

The effective free energy for a single Gaussian chain with repulsive slip-links can be expressed as the single chain version of Eq. (27),

$$
\mathcal{F}_{\text {eff }}\left(\left\{\boldsymbol{R}_{k}\right\},\left\{N_{k}\right\}, Z\right)=\frac{3 k_{B} T}{2 b^{2}} \sum_{k=1}^{Z} \frac{\left(\boldsymbol{R}_{k}-\boldsymbol{R}_{k-1}\right)^{2}}{N_{k}} .
$$

Introducing the effective chemical potential for subchains, $\epsilon$, we define the following effective grand potential (grand canonical thermodynamic potential) for the repulsive slip-link model:

$$
\mathcal{J}_{\text {eff }}\left(\left\{\boldsymbol{R}_{k}\right\},\left\{N_{k}\right\}, Z\right) \equiv \mathcal{F}_{\text {eff }}\left(\left\{\boldsymbol{R}_{k}\right\},\left\{N_{k}\right\}, Z\right)-\epsilon Z
$$

The effective chemical potential $\epsilon$ is determined from the following condition for the equilibrium average number of subchains:

$$
\langle Z\rangle_{\mathrm{eq}} \equiv Z_{0}=\frac{N}{N_{0}},
$$

where $\langle\ldots\rangle_{\text {eq }}$ represents the equilibrium statistical average calculated by the equilibrium probability distribution. Here, $N$ is the number of segments in a chain and $N_{0}$ corresponds to the equilibrium average number of segments in a subchain.

All the equilibrium statistical properties can be calculated by the effective grand potential (Eq. (32)). The equilibrium statistical probability distribution is given as the standard 
Boltzmann distribution,

$$
\begin{aligned}
P_{\text {eq }}\left(\left\{\boldsymbol{R}_{k}\right\},\left\{N_{k}\right\}, Z\right)= & \frac{1}{\Xi \Lambda^{3(Z+1)} N^{Z-1}} \delta\left(N-\sum_{k=1}^{Z} N_{k}\right) \\
& \times \exp \left[-\frac{\mathcal{J}_{\text {eff }}\left(\left\{\boldsymbol{R}_{k}\right\},\left\{N_{k}\right\}, Z\right)}{k_{B} T}\right],
\end{aligned}
$$

where $\Lambda$ is the thermal de Broglie wavelength and $\Xi$ is the grand partition function. (The subscript "eq" in Eq. (34) refers to the equilibrium state.) The thermal de Broglie wavelength was introduced to make the grand partition function dimensionless. The delta function in Eq. (34) comes from the constraint that the total number of segments in a chain is constant. The grand partition function $\Xi$ is defined as

$$
\begin{aligned}
\Xi \equiv & \sum_{Z=1}^{\infty} \frac{1}{\Lambda^{3(Z+1)} N^{Z-1}} \int d\left\{\boldsymbol{R}_{k}\right\} d\left\{N_{k}\right\} \delta\left(N-\sum_{k=1}^{Z} N_{k}\right) \\
& \times \exp \left[-\frac{\mathcal{J}_{\text {eff }}\left(\left\{\boldsymbol{R}_{k}\right\},\left\{N_{k}\right\}, Z\right)}{k_{B} T}\right] .
\end{aligned}
$$

Here, we introduced the shorthand notation for the integrals over $\left\{\boldsymbol{R}_{k}\right\}$ and $\left\{N_{k}\right\}$,

$$
\int d\left\{\boldsymbol{R}_{k}\right\} d\left\{N_{k}\right\} \equiv \prod_{k=0}^{Z} \int d \boldsymbol{R}_{k} \prod_{k=1}^{Z} \int_{0}^{N} d N_{k} .
$$

By calculating the integrals over $\left\{\boldsymbol{R}_{k}\right\}$, Eq. (35) becomes as follows:

$$
\begin{aligned}
\Xi= & \frac{\mathcal{V}}{\Lambda^{3}} \sum_{Z=1}^{\infty}\left[\left(\frac{2 \pi N b^{2}}{3 \Lambda^{2}}\right)^{3 / 2} e^{\epsilon / k_{B} T}\right]^{Z} \frac{1}{N^{5 Z / 2-1}} \\
& \times \int d\left\{N_{k}\right\} \delta\left(N-\sum_{k=1}^{Z} N_{k}\right) \prod_{k=1}^{Z} N_{k}^{3 / 2},
\end{aligned}
$$

where $\mathcal{V}$ is the volume of the system. The integrals over $\left\{N_{k}\right\}$ in Eq. (37) can analytically be calculated as

$$
\frac{1}{N^{5 Z / 2-1}} \int d\left\{N_{k}\right\} \delta\left(N-\sum_{k=1}^{Z} N_{k}\right) \prod_{k=1}^{Z} N_{k}^{3 / 2}=\frac{(3 \sqrt{\pi} / 4)^{Z}}{\Gamma(5 Z / 2)},
$$

where $\Gamma(x)$ is the gamma function. ${ }^{25}$ The detailed calculation is described in Appendix B 1. By substituting Eq. (38) into Eq. (37), finally we have the following expression:

$$
\begin{aligned}
\Xi & =\frac{\mathcal{V}}{\Lambda^{3}} \sum_{Z=1}^{\infty} \frac{1}{\Gamma(5 Z / 2)}\left[\frac{3 \pi^{2}}{4}\left(\frac{2 N b^{2}}{3 \Lambda^{2}}\right)^{3 / 2} e^{\epsilon / k_{B} T}\right]^{Z} \\
& =\frac{\mathcal{V}}{\Lambda^{3}} \xi E_{5 / 2,5 / 2}(\xi) .
\end{aligned}
$$

Here, we defined the dimensionless effective fugacity $\xi$ as

$$
\xi \equiv \frac{3 \pi^{2}}{4}\left(\frac{2 N b^{2}}{3 \Lambda^{2}}\right)^{3 / 2} e^{\epsilon / k_{B} T}
$$

$\xi$ is determined to satisfy the condition for $\langle Z\rangle_{\text {eq }}$ (Eq. (33)). $E_{\alpha, \beta}(x)$ in the last line of Eq. (39) is the (generalized) Mittag-
Leffler function ${ }^{26}$ defined as

$$
E_{\alpha, \beta}(x) \equiv \sum_{n=0}^{\infty} \frac{x^{n}}{\Gamma(\alpha n+\beta)} .
$$

Unfortunately, the Mittag-Leffler function is not easy to handle. Therefore, in Secs. III C-III E, we derive simple and approximate expressions rather than exact expressions.

\section{B. Slip-linked subchain number distribution}

First, we calculate the slip-linked subchain number distribution. The distribution function of the subchain number $Z$ can be expressed as follows:

$$
P_{\mathrm{eq}}(Z)=\int d\left\{\boldsymbol{R}_{k}\right\} d\left\{N_{k}\right\} P_{\mathrm{eq}}\left(\left\{\boldsymbol{R}_{k}\right\},\left\{N_{k}\right\}, Z\right) .
$$

By integrating Eq. (42) over $\left\{\boldsymbol{R}_{k}\right\}$ and $\left\{N_{k}\right\}$, we have the following expression:

$$
P_{\mathrm{eq}}(Z)=\frac{\xi^{Z-1}}{\Gamma(5 Z / 2) E_{5 / 2,5 / 2}(\xi)} .
$$

Equation (43) cannot be expressed by elementary functions. This is in contrast to the non-interacting slip-link model, of which subchain number distribution is simply given as a Poisson distribution (Eq. (A7) in Appendix A).

We can utilize the saddle point approximation to obtain a simple approximate form for Eq. (43) (see Appendix C 1),

$$
P_{\mathrm{eq}}(Z) \approx \sqrt{\frac{5 Z_{0}+1}{4 \pi Z_{0}^{2}}} \exp \left[-\frac{5 Z_{0}+1}{4 Z_{0}^{2}}\left(Z-Z_{0}\right)^{2}\right] .
$$

Figure 1 shows the subchain number distribution functions for various values of $Z_{0}$ by Eq. (43) (exact) and Eq. (44) (approximation). For comparison, the distribution function of the non-interaction slip-link model (Eq. (A7) in Appendix A) is also shown in Figure 1. We find that the approximate form (Eq. (44)) works very well even for relatively small $Z_{0}$ such as $Z_{0}=5$.

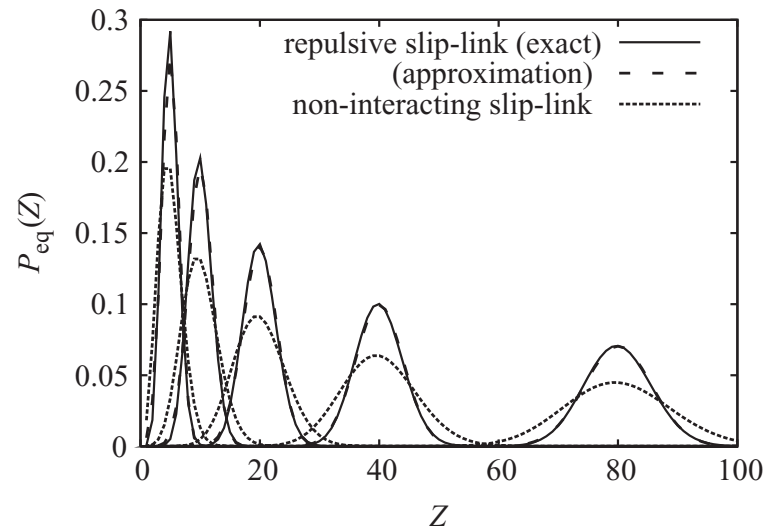

FIG. 1. The slip-linked subchain number distribution functions with various values of $Z_{0}$. Solid and dashed curves show the exact and approximate distribution functions for the single chain repulsive slip-link model, respectively. The dotted curves show the Poisson distribution which corresponds to the distribution of the single chain non-interacting slip-link model. $Z_{0}=5,10,20$, 40 , and 80 from left to right. The approximate distributions almost coincide with the exact distributions. 


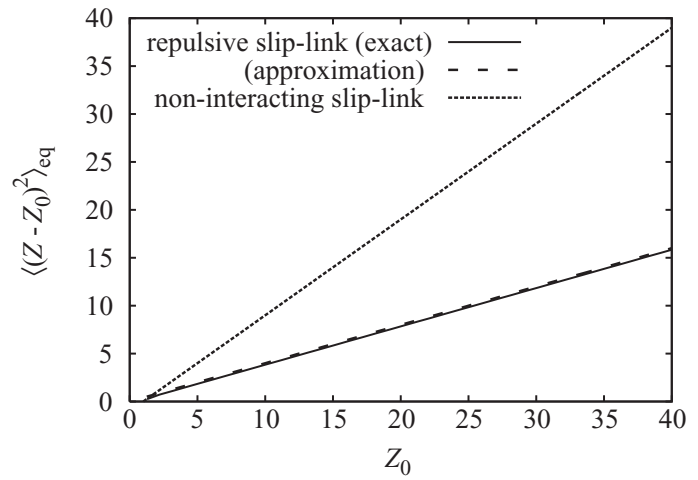

FIG. 2. Dependence of the variance of slip-linked subchain number $\left\langle\left(Z-Z_{0}\right)^{2}\right\rangle_{\text {eq }}$ on the average $\langle Z\rangle_{\text {eq }}=Z_{0}$. The solid and dashed curves show the exact and approximate variances for the repulsive slip-link model, and the dotted curve shows the variance for the non-interacting slip-link model $\left(\left\langle\left(Z-Z_{0}\right)^{2}\right\rangle_{\text {eq }}=Z_{0}-1\right)$. The approximate curve is very close to the exact curve.

The relation between the average and variance of $Z$ is easily obtained from Eq. (44),

$$
\left\langle\left(Z-Z_{0}\right)^{2}\right\rangle_{\mathrm{eq}} \approx \frac{2 Z_{0}^{2}}{5 Z_{0}+1} \approx \frac{2}{5} Z_{0} \quad\left(\text { for } Z_{0} \gg 1\right) .
$$

Figure 2 shows the relation between $\langle Z\rangle_{\mathrm{eq}}=Z_{0}$ and $\left\langle\left(Z-Z_{0}\right)^{2}\right\rangle_{\text {eq }}$, calculated by the exact and approximate forms. Again, we find that the approximate form (Eq. (44)) works well even for relatively small $Z_{0}$. For sufficiently large $Z_{0}$, we find that the variance of the repulsive slip-link model is smaller than the one for non-interacting slip-link model by the factor 2/5 (see Eq. (A8) in Appendix A). The distribution of $Z$ of the repulsive slip-link model is sharper than the one of the non-interacting slip-link model, as shown in Figure 1.

\section{Segment number distribution}

Second, we calculate the distribution function of the segment number in a subchain. We express the number of segments as $n$. The segment number distribution function $P_{\mathrm{eq}}(n)$ is expressed as follows:

$$
\begin{aligned}
P_{\mathrm{eq}}(n)= & \sum_{Z=1}^{\infty} \int d\left\{\boldsymbol{R}_{k}\right\} d\left\{N_{k}\right\} \\
& \times\left[\frac{1}{Z} \sum_{l=1}^{Z} \delta\left(n-N_{l}\right)\right] P_{\mathrm{eq}}\left(\left\{\boldsymbol{R}_{k}\right\},\left\{N_{k}\right\}, Z\right) .
\end{aligned}
$$

By calculating the integrals over $\left\{\boldsymbol{R}_{k}\right\}$ in Eq. (46), we have

$$
\begin{aligned}
P_{\mathrm{eq}}(n)= & \frac{\mathcal{V}}{\Xi \Lambda^{3} N} \sum_{Z=1}^{\infty} \frac{\xi^{Z}}{N^{5 Z / 2-2}} \int d\left\{N_{k}\right\}\left[\frac{1}{Z} \sum_{l=1}^{Z} \delta\left(n-N_{l}\right)\right] \\
& \times \delta\left(N-\sum_{k=1}^{Z} N_{k}\right) \prod_{k=1}^{Z} N_{k}^{3 / 2}
\end{aligned}
$$

Integrals over $\left\{N_{k}\right\}$ in Eq. (47) can be calculated analytically. For $Z \geq 2$, we have

$$
\begin{gathered}
\frac{1}{N^{5 Z / 2-2}} \int d\left\{N_{k}\right\}\left[\frac{1}{Z} \sum_{l=1}^{Z} \delta\left(n-N_{l}\right)\right] \delta\left(N-\sum_{k=1}^{Z} N_{k}\right) \prod_{k=1}^{Z} N_{k}^{3 / 2} \\
=\left(\frac{n}{N}\right)^{3 / 2}\left(1-\frac{n}{N}\right)^{5(Z-1) / 2-1} \frac{(3 \sqrt{\pi} / 4)^{Z-1}}{\Gamma(5(Z-1) / 2)}
\end{gathered}
$$

(see Appendix B 1), and for $Z=1$ we have

$$
\frac{1}{N^{1 / 2}} \int_{0}^{N} d N_{1} \delta\left(n-N_{1}\right) \delta\left(N-N_{1}\right) N_{1}^{3 / 2}=\delta\left(\frac{n}{N}-1\right) .
$$

Thus, Eq. (47) can be rewritten as follows:

$$
\begin{aligned}
P_{\mathrm{eq}}(n)= & \frac{4}{3 \sqrt{\pi} N E_{5 / 2,5 / 2}(\xi)}\left[\delta\left(\frac{n}{N}-1\right)+\sum_{Z=2}^{\infty}\left(\frac{n}{N}\right)^{3 / 2}\right. \\
& \left.\times\left(1-\frac{n}{N}\right)^{5(Z-1) / 2-1} \frac{\xi^{Z-1}}{\Gamma(5(Z-1) / 2)}\right] .
\end{aligned}
$$

As before, we can obtain a simple approximate expression by using the saddle point approximation. (See Appendix C 2.) For sufficiently large $Z_{0}$, Eq. (48) is approximated as

$$
P_{\mathrm{eq}}(n) \approx \frac{25}{6} \sqrt{\frac{10}{\pi}} \frac{n^{3 / 2}}{N_{0}^{5 / 2}} \exp \left(-\frac{5 n}{2 N_{0}}\right) .
$$

Equation (51) is qualitatively different from one of the single chain non-interacting slip-link model (Eq. (A12) in Appendix A). Figure 3 shows the segment number distribution functions for repulsive and non-interacting slip-link models, for sufficiently large $Z_{0}$. The distribution functions are normalized to satisfy the following normalization condition:

$$
\int_{0}^{\infty} d\left(n / N_{0}\right) P_{\text {eq }}\left(n / N_{0}\right)=1
$$

We can observe differences between two models clearly. The difference is especially large for small $n$. Equation (51) is not a monotonically decreasing function and it has a maximum at $n / N_{0}=3 / 5$. Also, Eq. (51) approaches to 0 at the limit of $n \rightarrow 0$, while the distribution function of the non-interacting slip-link model approaches to a non-zero constant. Intuitively,

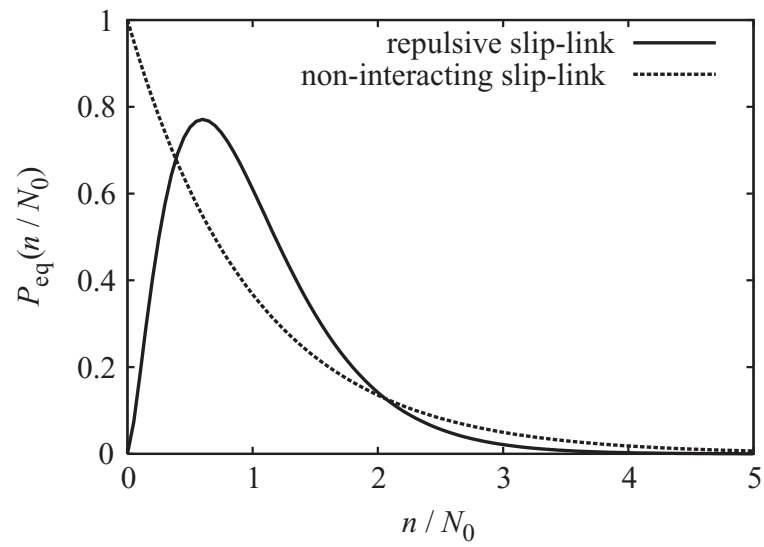

FIG. 3. The segment number distribution functions for sufficiently large $Z_{0}$. Solid and dotted curves show the distribution functions for the repulsive and non-interacting slip-link models, respectively. 
this can be understood as the effect of the strong short-range repulsion between the two neighboring slip-links.

\section{Bond vector and bond length distributions}

Finally, we calculate the distribution functions for the bond vector and bond length. Here, we define the bond vector as the vector which connects the two successive slip-links (or pair of a slip-link and a chain end) on the same chain. If we express the bond vector as $\boldsymbol{Q}$, the bond vector distribution function $P_{\mathrm{eq}}(\boldsymbol{Q})$ is expressed as follows:

$$
\begin{aligned}
P_{\mathrm{eq}}(\boldsymbol{Q})= & \sum_{Z=1}^{\infty} \int d\left\{\boldsymbol{R}_{k}\right\} d\left\{N_{k}\right\} \\
& \times\left[\frac{1}{Z} \sum_{l=1}^{Z} \delta\left(\boldsymbol{Q}-\boldsymbol{R}_{l}+\boldsymbol{R}_{l-1}\right)\right] P_{\mathrm{eq}}\left(\left\{\boldsymbol{R}_{k}\right\},\left\{N_{k}\right\}, Z\right) .
\end{aligned}
$$

Equation (53) can be modified as follows:

$$
\begin{aligned}
P_{\mathrm{eq}}(\boldsymbol{Q})= & \frac{\mathcal{V}}{\Xi \Lambda^{3}}\left(\frac{3}{2 \pi b^{2}}\right)^{3 / 2} \frac{1}{N} \int_{0}^{N} d n\left(\frac{N}{n}\right)^{3 / 2} e^{-3 \boldsymbol{Q}^{2} / 2 n b^{2}} \\
& \times \sum_{Z=1}^{\infty} \frac{\xi^{Z}}{N^{5 Z / 2-2}} \int d\left\{N_{k}\right\} \prod_{k=1}^{Z} N_{k}^{3 / 2} \\
& \times\left[\frac{1}{Z} \sum_{l=1}^{Z} \delta\left(n-N_{l}\right)\right] \delta\left(N-\sum_{k=1}^{Z} N_{k}\right)
\end{aligned}
$$

The integrals over $\left\{N_{k}\right\}$ can be calculated in the same way as the case of the segment number distribution function. Then, we have

$$
P_{\mathrm{eq}}(\boldsymbol{Q})=\left(\frac{3}{2 \pi b^{2}}\right)^{3 / 2} \int_{0}^{N} d n \frac{1}{n^{3 / 2}} e^{-3 \boldsymbol{Q}^{2} / 2 n b^{2}} P_{\mathrm{eq}}(n) .
$$

For sufficiently large $Z_{0}$, the bond vector distribution function can be approximately expressed as follows, by using Eq. (51):

$$
\begin{aligned}
P_{\mathrm{eq}}(\boldsymbol{Q}) \approx & \frac{25}{6} \sqrt{\frac{10}{\pi}}\left(\frac{3}{2 \pi N_{0} b^{2}}\right)^{3 / 2} \frac{1}{N_{0}} \\
& \times \int_{0}^{\infty} d n \exp \left(-\frac{3 \boldsymbol{Q}^{2}}{2 n b^{2}}-\frac{5 n}{2 N_{0}}\right) \\
= & \frac{75}{2 \pi^{2}} \frac{|\boldsymbol{Q}|}{N_{0}^{2} b^{4}} K_{1}\left(\sqrt{\frac{15}{N_{0} b^{2}}}|\boldsymbol{Q}|\right),
\end{aligned}
$$

where $K_{1}(x)$ is the first-order modified Bessel function of the second kind. ${ }^{25}$ (The detailed calculation is described in Appendix B 2.) From Eq. (56), the bond length distribution function (the bond length $Q$ is defined as $Q \equiv|\boldsymbol{Q}|$ ) becomes

$$
P_{\mathrm{eq}}(Q)=4 \pi Q^{2} P_{\mathrm{eq}}(\boldsymbol{Q}) \approx \frac{150}{\pi} \frac{Q^{3}}{N_{0}^{2} b^{4}} K_{1}\left(\sqrt{\frac{15}{N_{0} b^{2}}} Q\right) .
$$

From Eq. (57), we find that the form of the bond length distribution function is also different from one of the single chain non-interacting slip-link model (Eq. (A15) in Appendix A). Figure 4 shows the bond length distribution functions for repulsive and non-interacting slip-link models, for sufficiently

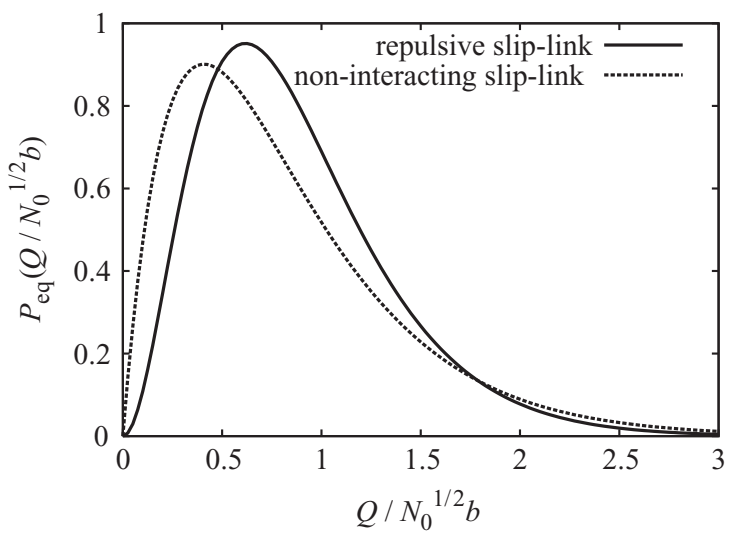

FIG. 4. The bond length distribution functions for sufficiently large $Z_{0}$. Solid and dotted curves show the distribution functions for the repulsive and noninteracting slip-link models, respectively.

large $Z_{0}$. The distribution functions are normalized to satisfy the following normalization condition:

$$
\int_{0}^{\infty} d\left(Q / \sqrt{N_{0} b^{2}}\right) P_{\mathrm{eq}}\left(Q / \sqrt{N_{0} b^{2}}\right)=1 .
$$

For small $Q$, the asymptotic form of Eq. (57) becomes

$$
P_{\mathrm{eq}}(Q) \approx \frac{10 \sqrt{15}}{\pi} \frac{Q^{2}}{N_{0}^{3 / 2} b^{3}} \propto Q^{2} \quad\left(\text { for } Q \ll \sqrt{N_{0} b^{2}}\right) .
$$

This dependence of the asymptotic form on $Q$ is different from the one for the non-interacting slip-link model $\left(P_{\text {eq }}(Q) \propto Q\right)$. The difference between two distributions is especially large for small $Q$. This trend is similar to the difference between the segment number distribution functions. The position of the maximum of Eq. (57) is $Q / \sqrt{N_{0} b^{2}}$ $\approx 0.616$, whereas it is $Q / \sqrt{N_{0} b^{2}}=1 / \sqrt{6} \approx 0.408$ for the non-interacting slip-link model. As before, these properties can be understood as the effect of the strong short-range repulsion between slip-links.

\section{E. Comparison with PCN simulations}

The distribution functions obtained in preceding subsections (Secs. III B-III D) are based on the single subchain approximation. However, the validity of the single chain approximation is not guaranteed for the PCN model. In this subsection, we perform PCN simulations with original and modified dynamic equations and directly calculate the distribution functions.

The simulations are performed for linear monodisperse polymers with $Z_{0}=5,10,20$, and 40 . The osmotic terms to prevent aggregation of slip-links ${ }^{2,10}$ are added to Eqs. (2), (4), and (17). The network reconstruction is used to realize the steady state (that is the equilibrium state for the modified model). The employed rule was NR1 in Ref. 10 (which is briefly explained in Sec. II A). The unit cell dimension for periodic boundary condition is $12 \sqrt{N_{0} b^{2}}$ and the total bond number in the cell is $\sim 10 \times 12^{3}$. The distribution functions are obtained for 10 independent simulations and the averages are reported here for noise reduction purposes. 


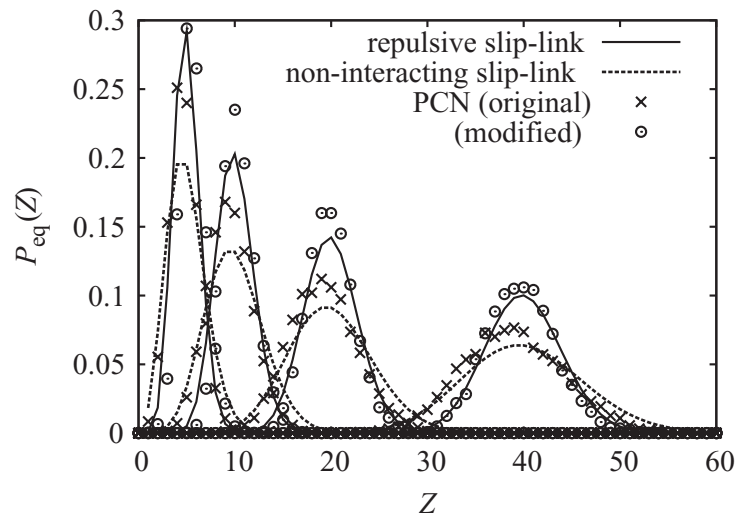

FIG. 5. The slip-linked subchain number distribution functions by single chain models and PCN simulations. Curves are theoretical predictions by single chain models and symbols are simulation data. $Z_{0}=5,10,20$, and 40 from left to right.

Figure 5 shows the comparison of the subchain number distribution functions $P_{\text {eq }}(Z)$ calculated by the single chain models and the PCN simulations. As shown in Figure 5, the data of the modified PCN model agree well with the repulsive slip-link model. The original PCN model gives a subchain number distribution function which is broader than the repulsive slip-link model but still sharper than the non-interacting slip-link model. Considering the roughness of the single chain approximation, the agreement between the modified PCN model and the repulsive slip-link model is rather surprising. The CReTA primitive path extraction by Tzoumanekas and Theodorou ${ }^{9}$ also shows subchain number distributions which are sharper than the Poissonian, but still broader than Eq. (44). Thus, these models will be in between the non-interacting slip-link model and the repulsive slip-link model (if we assume that the repulsive interaction between slip-links is the main reason which causes non-Poissonian distribution).

Figure 6 shows the comparisons of the segment number distribution functions $P_{\mathrm{eq}}(n)$ for $Z_{0}=40$. (The sharp peaks of $P_{\text {eq }}(n)$ by the PCN simulations around $n / N_{0}=0.1$ are the artifacts due to the cutoff of the segment number. ${ }^{10}$ In the followings, we neglect these artificial peaks.) Unlike the case of the subchain number distribution, we can observe the deviation of the modified PCN model data from the repulsive slip-link model. As shown in Figure 6, the segment number distribution function of the modified PCN model is somehow shifted to large $n$ region. However, the asymptotic behavior of the modified PCN model at small $n$ is consistent with the repulsive slip-link model $\left(P_{\mathrm{eq}}(n) \propto n^{3 / 2}\right)$. The original PCN model gives the distribution between the non-interacting and the repulsive slip-link models. This seems to be qualitatively similar to the case of the subchain number distribution. Here it is worth mentioning that the CReTA primitive path extraction data ${ }^{9}$ gives the qualitatively similar asymptotic behavior, $P(n) \rightarrow 0$ at $n \rightarrow 0$, to the modified PCN model and the repulsive slip-link model. We will discuss the segment number distribution functions later in detail (Sec. IV B).

Figure 7 shows the bond length distribution functions $P_{\text {eq }}(Q)$ for $Z_{0}=40$. We can find the bond length distribution function of the modified PCN model deviates from the

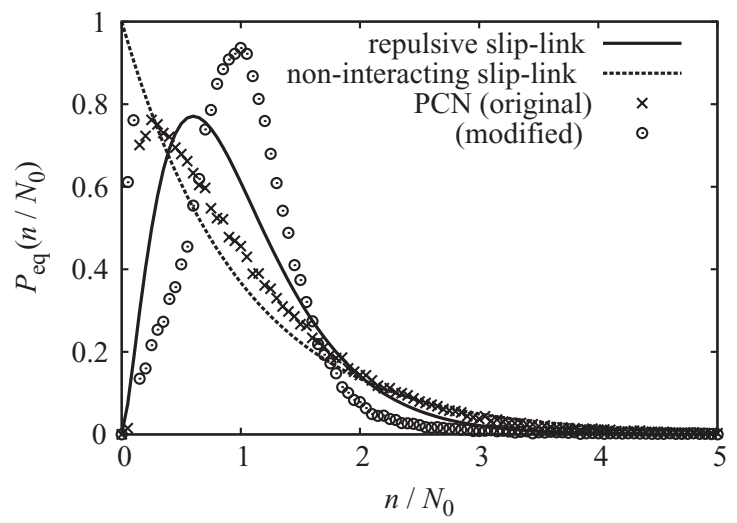

FIG. 6. The segment number distribution functions calculated by single chain models and PCN simulations (for $Z_{0}=40$ ). Curves and symbols represent the results by single chain models and PCN simulations, respectively.

repulsive slip-link model. This result is physically natural because the bond length distribution function and the segment number distribution function are expected to be related each other. In the single chain model, actually their relation is simply expressed by Eq. (55). The bond length distribution function of the modified PCN model is slightly shifted to large $Q$ region. The asymptotic behavior of the modified PCN model at small $Q$ is also consistent with the repulsive slip-link model $\left(P_{\mathrm{eq}}(Q) \propto Q^{2}\right)$. The original PCN model data are again between the non-interacting and repulsive slip-link models.

We can observe that both the original and modified PCN simulation data have relatively large probability for large $Q$. This implies that subchains in the system are stretched compared with the ideal Gaussian subchains. This would be because chains are required to form a connected, tetra-functional network structure in the PCN model. (In the PCN model, a slip-link spatially binds two chains, and the bound chains will be somehow stretched.) This can be interpreted as a result of the interchain force-balance condition imposed in the PCN model (this condition is automatically imposed by Eqs. (1) and (2)). The possible chain conformations are limited compared with the single chain model, where no such constraint exists. As a result, the bond length distribution function becomes broader and deviates from the distribution

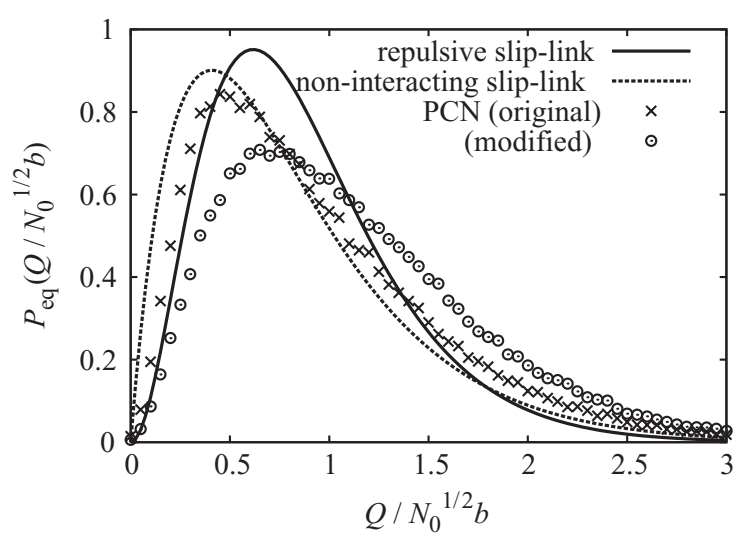

FIG. 7. The bond length distribution functions calculated by single chain models and PCN simulations (for $Z_{0}=40$ ). Curves and symbols represent the results by single chain models and PCN simulations, respectively. 
function in the single chain model. This is qualitatively consistent with the simulation result. Also, the segment number distribution would shift to large segment number direction, which is again qualitatively consistent with the simulation result. Therefore, we expect the constraint for chains to be the origin of the deviation of $P_{\mathrm{eq}}(Q)$. The origin of the deviation of $P_{\text {eq }}(n)$ will also be the same.

In the single chain model, effects of the surrounding chains are expressed only by the effective chemical potential $\epsilon$. Such a rough approximation would not be fully applicable to describe the statistics of slip-linked multi chains. The effective chemical potential controls only the number of slip-linked subchains, and the statistics of bond vectors or segment numbers are determined only by the effective free energy. If interactions between the target chain and the surrounding chains are not simple and cannot be described by a single parameter $\epsilon$, the single chain model cannot reproduce the PCN simulation results. To analyze the statistical properties of the PCN model theoretically, therefore, we will need to improve the single chain approximation to take account of the effects of surrounding chains. For example, some sort of spatial correlation effect may be took into account to the model, by using the connectivity information (such as the connectivity map $C$ in the PCN model). Several radial distribution functions of nodes are obtained by the CReTA primitive path extraction. ${ }^{9}$ Kindt and Briels ${ }^{21}$ modeled that the average number of entanglements between two chains as a function of the distance between centers of mass of chains for their highly coarsegrained single particle model of entangled polymers. If we use the connectivity information and reproduce these spatial correlations, several statistical properties may be improved. Of course, there are other possible factors which affect the statistical properties of the PCN model. (As we mentioned, the network reconstruction rule ${ }^{10}$ affects several statistical properties of the PCN model. Recent simulation results show that the spatial correlation between nodes is somewhat affected by the form of the interaction potential between nodes, although rheological properties are almost unchanged. ${ }^{27}$ Such a local structural change may affect the distribution functions to some extent. If we directly control the spatial correlations of slip-links by controlling the reconstruction rules and the connectivity information, the distribution functions will be affected.)

\section{DISCUSSION}

\section{A. Detailed balance condition and effective free energy}

As shown in Sec. II B, the PCN dynamic equations can be reduced to the detailed-balanced Langevin equations with two modifications. It is rather surprising that simple modifications can reproduce rather complicated, detailed-balanced Langevin equations. The modified (detailed-balanced) version of the PCN model can reproduce several probability distribution functions which are qualitatively similar to the ones predicted by the single chain repulsive slip-link model.

Here, we consider the original PCN dynamic equation for $\left\{N_{i, k}\right\}$ (Eqs. (3) and (4)) again. The fact that we only need relatively minor modifications for the original PCN dynamics equations implies that the original PCN dynamics itself is already a reasonable approximation for a statistical mechanically sound model. Actually, we can show that the original PCN model approximately satisfies the detailed balance condition under a certain condition. We consider the most probable value of $N_{i, k}$ under a given $\left\{\boldsymbol{R}_{i, k}\right\}$ and $Z_{i}$. The most probable state $\left\{\bar{N}_{i, k}\right\}$ minimizes the effective free energy (Eq. (27)). $\bar{N}_{i, k}$ is simply given as

$$
\bar{N}_{i, k}=\bar{\rho}_{i}\left|\boldsymbol{R}_{i, k}-\boldsymbol{R}_{i, k-1}\right|,
$$

where $\bar{\rho}_{i}$ is the most probable value of the monomer density along the chain on the $i$ th chain $\left(\bar{\rho}_{i, k}\right.$ is independent of $k$, and thus, we simply describe it as $\bar{\rho}_{i}$ ). If the deviation of $N_{i, k}$ from the most probable value is small, we can rewrite $N_{i, k}$ as $N_{i, k}=\bar{N}_{i, k}+\delta N_{i, k}$ and expand the free energy or the dynamic equation into power series of $\left\{\delta N_{i, k}\right\}$. Expanding Eqs. (25), (22), and (27) into power series and retaining only the leading order terms, we have

$$
\begin{gathered}
\mathcal{F}_{\text {eff }}\left(\left\{\boldsymbol{R}_{i, k}\right\},\left\{N_{i, k}\right\},\left\{Z_{i}\right\}\right) \\
\approx \frac{3 k_{B} T}{2 b^{2}} \sum_{i} \sum_{k=1}^{Z_{i}}\left[\frac{\left(\boldsymbol{R}_{i, k}-\boldsymbol{R}_{i, k-1}\right)^{2}}{\bar{N}_{i, k}}+\frac{\delta N_{i, k}^{2}}{\bar{\rho}_{i}^{2} \bar{N}_{i, k}}\right] \\
\rho_{i, k}\left(\left\{\boldsymbol{R}_{i, k}\right\},\left\{N_{i, k}\right\}\right) \approx \bar{\rho}_{i} \\
J_{i, k}(t) \approx \frac{2 \bar{\rho}_{i}^{2}}{\zeta} \frac{3 k_{B} T}{b^{2}}\left[\frac{\delta N_{i, k}}{\bar{\rho}_{i}^{2} \bar{N}_{i, k}}-\frac{\delta N_{i, k+1}}{\bar{\rho}_{i}^{2} \bar{N}_{i, k+1}}\right]+\sqrt{\frac{4 \bar{\rho}_{i}^{2} k_{B} T}{\zeta}} w_{i, k}^{(N)}(t) .
\end{gathered}
$$

The original PCN model, Eqs. (4) and (5) also reduce to Eqs. (62) and (63), respectively. Therefore, the original PCN model satisfies the detailed balance condition, if $\left\{N_{i, k}\right\}$ is in the vicinity of the most probable state $\left\{\bar{N}_{i, k}\right\}$. (Intuitively, this is because the segment density $\rho_{i, k}$ is not sensitive to the node index $k$ in the vicinity of the most probable state. The harmonic and arithmetic averages are almost the same and the spurious drift velocity is negligibly small, under this condition.) Unfortunately, this condition is not always satisfied even in equilibrium, because $\delta N_{i, k}$ is generally not negligibly small due to the thermal fluctuation. Nevertheless, the probability that $\left\{N_{i, k}\right\}$ is close to $\left\{\bar{N}_{i, k}\right\}$ is expected not to be small. Thus, we consider that the analysis performed here qualitatively explains why the original PCN model works as a good approximation.

The new definition for the segment density $\rho_{i, k}$ (Eq. (22)) proposed in this work is expressed as the harmonic average of the local densities in two neighboring bonds. Although this form is not intuitive, from the view point of the discretization scheme, it is not unreasonable. The PCN dynamic equation for $\left\{N_{i, k}\right\}$ (Eqs. (3) and (4)) is interpreted as the stochastic diffusion equation on one-dimensional discrete lattice. At the continuum limit, the PCN dynamic equation will reduce to the stochastic partial differential equation ${ }^{28}$ which contains the multiplicative noise. To discretize the multiplicative noises appear in the stochastic partial differential equations, non-arithmetic averages are sometimes preferred than the 
simple arithmetic average. For example, the multiplicative noise in the dynamic density functional equation can accurately be discretized with the geometric average form. ${ }^{29}$ (The geometric or harmonic average is zero, if one of the averaged numbers is zero, whereas the arithmetic average is nonzero. Such a property is required to generate the multiplicative noise accurately and stably.) Thus, we expect that the harmonic average form is more natural than the arithmetic average form.

We should note that the statistical properties of the PCN network reconstruction rules ${ }^{10,17}$ are not considered in this work. The network reconstruction rules proposed so far do not satisfy the detailed balance condition, and therefore, the PCN model is not fully detailed-balanced, even if we employ the modified dynamic equations. (Especially for small $Z_{0}$, the network reconstruction rule affects the statistical properties significantly.) To understand the statistical properties of the full PCN dynamics, the statistical mechanical analysis of the network reconstruction rules is also required. It seems to be difficult to satisfy the detailed balance condition in the network reconstruction process of multi chain slip-link models due to its intrinsic complicatedness, and thus, it is left for a future work. (The exact equilibrium statistics of a slip-linked multi chain system is not simple nor intuitive ${ }^{16}$ unlike a single chain system.) Although we do not consider further modifications here, the current work will be useful to find strategies for other modifications which improve the PCN model.

\section{B. Segment number distribution}

As we showed and discussed in Secs. II and III, the effective repulsive interaction between slip-links (Eq. (30)) affects statistical properties of the network qualitatively. We expect that the statistics can further be changed by changing the interaction potential (or interaction strength) between slip-links. In fact, if we consider the limit of the strong repulsion, distribution functions become much different from noninteracting slip-link model (see Appendix D). Roughly speaking, the variance of the subchain number distribution function can be used to estimate the strength of the effective repulsive interaction between slip-links. It is interesting to compare our model (or our picture) with other models or primitive path extraction results, and discuss whether our model is similar to others or not.

Among the examined distribution functions, the segment number distribution function seems to be the most sensitive to the interaction between slip-links. The segment number distribution of the single chain repulsive slip-link model is qualitatively much different from that of the single chain noninteracting slip-link model. The segment number distribution functions which have similar forms to Eq. (51) are already proposed by Tzoumanekas and Theodorou, ${ }^{9}$ and Greco. ${ }^{30}$

Here, we compare our model with these distribution functions. Tzoumanekas and Theodorou proposed the following empirical form for the segment number distribution, to fit their CReTA primitive path extraction data:

$$
P_{\mathrm{eq}}(n)=\frac{1}{N_{0}} \frac{\tilde{b} \tilde{c}}{\tilde{c}-\tilde{b}}\left(e^{-\tilde{b} n / N_{0}}-e^{-\tilde{c} n / N_{0}}\right)
$$

Here $1<\tilde{b}<2$ is a fitting parameter and $\tilde{c}=\tilde{b} /(\tilde{b}-1)$. They reported that the segment number distribution functions obtained by the CReTA can be fitted well to Eq. (64) with $\tilde{b}=1.30$. Equation (64) can also be utilized to fit the distribution functions by the original PCN model with the fitting parameter $\tilde{b}=1.96 .{ }^{10}$ This value is close to 2 , and for such a case, Eq. (64) can be approximated well by the following simple form:

$$
P_{\mathrm{eq}}(n) \rightarrow \frac{4 n}{N_{0}^{2}} e^{-2 n / N_{0}} \quad(\tilde{b} \rightarrow 2) .
$$

Equation (65) is quite similar to Eq. (51). Tzoumanekas and Theodorou proposed that the physical origin of such a nonmonotonic form of Eq. (64) is the effective repulsion (or the blocking effect) between topological constraints, which depends on the chemical distance. This is qualitatively the same as our repulsive slip-link model. Of course, the CReTA primitive path extraction results and their molecular model are different from the PCN model and our slip-link model. For example, the CReTA primitive path data are taken for an atomistic polymer model, while the PCN model employs a rather coarse-grained Gaussian chain model. Besides, it is not clear whether the slip-links in the PCN model and the topological constraints extracted by the CReTA have the same physical properties. Thus, the distribution function by the PCN model does not necessarily coincide with the one by the CReTA. Actually, at least the values of the fitting parameter $\tilde{b}$ for two distributions are different. Nonetheless, we consider that the similarity between the Tzoumanekas-Theodorou model and our repulsive slip-link model implies that there are some common properties of slip-links (or topological constraints). (We expect that the detailed form of the effective repulsive potential between topological constraints in the TzoumanekasTheodorou model is different from our repulsive slip-link model. Judging from the distribution functions, the repulsive interaction in their model seems to be weaker than our model. But, the detail repulsion mechanisms in the TzoumanekasTheodorou model or the CReTA results are not so clear. Further investigations will be future works.)

On the other hand, in the Greco model, the segment number distribution function is given as

$$
\begin{aligned}
P_{\mathrm{eq}}(n) \propto & s(\tilde{m}) \sqrt{\frac{n}{N_{0}}} e^{-\tilde{m}^{2} n / N_{0}}+\sqrt{\pi}\left[\frac{1}{2}+s^{2}(\tilde{m}) \frac{n}{N_{0}}\right] \\
& \times e^{\left(1 / \tilde{m}^{2}-2\right) n / N_{0}} \operatorname{erfc}\left(-s(\tilde{m}) \sqrt{\frac{n}{N_{0}}}\right),
\end{aligned}
$$

where $\tilde{m} \geq 1$ is the renormalized chemical potential (which is used as a fitting parameter), $s(x) \equiv\left(x^{2}-1\right) / x$, and $\operatorname{erfc}(x)$ is the complementary error function. ${ }^{25}$ Equation (66) has a similar form to Eq. (64). ${ }^{10,30}$ In the Greco model, the slip-links are non-interacting. The non-monotonic nature of Eq. (66) comes from the fluctuation effect due to the smallness of the system. This is qualitatively different from our model. It is interesting that different mechanisms (the fluctuation effect and the repulsive interaction between slip-links) give the similar result. Further progress of theories would be required to understand the origin of non-monotonic segment number distribution functions. 
J. Chem. Phys. 135, 184904 (2011)

Although there will be other factors (the osmotic force between nodes, the network reconstruction, or constraint to form the tetra-functional network structure), the interaction between slip-links is one important factor which determines the network statistics. The concept of the effective repulsive interaction between slip-links will also be useful to consider statistical properties of other models or methods, such as the primitive path extraction methods ${ }^{7-9}$ or other slip-link based models without explicit free energy model. ${ }^{31}$ For example, the differences among various primitive path extraction methods may be related to the differences of the effective slip-link interaction potential models. Once we obtain the explicit form of the effective interaction potential, it will be possible to tune it so that the model reproduces the required equilibrium statistics. We can employ the effective interaction potential for sliplinks determined from the network statistics by the primitive path extraction methods to make the slip-link models compatible with the primitive path extraction data. (We can easily replace the effective free energy model by other models to tune the statistics of the modified PCN model because the forces in the modified PCN model are expressed as variational forms.)

\section{CONCLUSIONS}

We showed that the PCN dynamic equations do not satisfy the detailed-balance condition, and therefore, the PCN model does not have the thermal equilibrium state. However, by introducing heuristic modifications, the $\mathrm{PCN}$ dynamics can be recovered to satisfy the detailed balance condition. We proposed two modifications. One is to change the definition of the segment density along the chain to the harmonic average form (Eq. (22)), and another is to add the spurious drift term to the monomer flux equation (Eq. (25)). From the modified PCN model, we obtained the effective free energy from which the equilibrium statistical properties of the PCN model are determined. The effective PCN free energy has a different form than the free energy of ideal Gaussian chains. This can be understood that the effective PCN free energy contains the contribution from the repulsive interaction between slip-links.

To analyze the equilibrium statistical properties of the PCN model, we constructed a single chain model with repulsive slip-links. The equilibrium distribution functions derived from the single chain repulsive slip-link model are qualitatively different from the ones for the single chain noninteracting slip-link model due to the repulsive interaction between slip-links. It was shown that the slip-linked subchain number distribution function for the repulsive slip-link model is much sharper than the one for non-interacting slip-links. The interaction between slip-links strongly affects the segment number distribution function, especially if the segment number is small. The equilibrium properties obtained from the single chain slip-link model are qualitatively similar to the PCN simulation results, although the agreement is not perfect. We consider the repulsive interaction between slip-links is an important factor which determines the statistical properties of the PCN model. The repulsive slip-link picture would be useful to improve the model statistics or design a new model.

\section{ACKNOWLEDGMENTS}

This work is supported by the JST-CREST.

\section{APPENDIX A: SINGLE CHAIN NON-INTERACTING SLIP-LINK MODEL}

In this appendix, we show a brief derivation of the equilibrium distribution functions for the single chain noninteracting slip-link model. ${ }^{24}$ (The derivation mainly follows Ref. 24, but the expressions are slightly modified to allow the direct comparison with the repulsive slip-link model in the main text.) The free energy of a Gaussian chain with noninteracting slip-links is expressed as the single chain version of Eq. (28),

$$
\begin{aligned}
\mathcal{F}_{0}\left(\left\{\boldsymbol{R}_{k}\right\},\left\{N_{k}\right\}, Z\right) \equiv & \frac{3 k_{B} T}{2 b^{2}} \sum_{k=1}^{Z} \frac{\left(\boldsymbol{R}_{k}-\boldsymbol{R}_{k-1}\right)^{2}}{N_{k}} \\
& +\frac{3 k_{B} T}{2} \sum_{k=1}^{Z} \ln N_{k} .
\end{aligned}
$$

As we mentioned in the main text, the Gaussian free energy contains a term which is proportional to $\ln N_{i}$, while the PCN effective free energy (Eq. (27)) does not.

The grand potential is defined as follows, by introducing the effective chemical potential for a subchain, $\epsilon_{0}$ :

$$
\mathcal{J}_{0}\left(\left\{\boldsymbol{R}_{k}\right\},\left\{N_{k}\right\}, Z\right) \equiv \mathcal{F}_{0}\left(\left\{\boldsymbol{R}_{k}\right\},\left\{N_{k}\right\}, Z\right)-\epsilon_{0} Z .
$$

The equilibrium statistical probability can be written as

$$
\begin{aligned}
P_{\mathrm{eq}}\left(\left\{\boldsymbol{R}_{k}\right\},\left\{N_{k}\right\}, Z\right)= & \frac{1}{\Xi_{0} \Lambda^{3(Z+1)} N^{Z-1}} \delta\left(N-\sum_{k=1}^{Z} N_{k}\right) \\
& \times \exp \left[-\frac{\mathcal{J}\left(\left\{\boldsymbol{R}_{k}\right\},\left\{N_{k}\right\}, Z\right)}{k_{B} T}\right]
\end{aligned}
$$

with $\Xi_{0}$ being the grand partition function. The grand partition function reduces to a simple form

$$
\begin{aligned}
\Xi_{0} \equiv & \sum_{Z=1}^{\infty} \frac{1}{\Lambda^{3(Z+1)} N^{Z-1}} \int d\left\{\boldsymbol{R}_{k}\right\} d\left\{N_{k}\right\} \delta\left(N-\sum_{k=1}^{Z} N_{k}\right) \\
& \times \exp \left[-\frac{\mathcal{J}\left(\left\{\boldsymbol{R}_{k}\right\},\left\{N_{k}\right\}, Z\right)}{k_{B} T}\right]=\frac{\mathcal{V}}{\Lambda^{3}} \xi_{0} e^{\xi_{0}} .
\end{aligned}
$$

Here, we defined the dimensionless effective fugacity $\xi_{0}$ as

$$
\xi_{0} \equiv e^{\epsilon_{0} / k_{B} T}\left(\frac{2 \pi b^{2}}{3 \Lambda^{2}}\right)^{3 / 2} .
$$

The slip-linked subchain number distribution function becomes the Poisson distribution,

$$
\begin{aligned}
P_{\mathrm{eq}}(Z) & =\int d\left\{\boldsymbol{R}_{k}\right\} d\left\{N_{k}\right\} P_{\mathrm{eq}}\left(\left\{\boldsymbol{R}_{k}\right\},\left\{N_{k}\right\}, Z\right) \\
& =\frac{1}{(Z-1) !} \xi_{0}^{Z-1} e^{-\xi_{0}} .
\end{aligned}
$$

Equation (A6) can be rewritten by using the relation, $\langle Z\rangle_{\mathrm{eq}}$ $=Z_{0}=\xi_{0}+1$,

$$
P_{\mathrm{eq}}(Z)=\frac{1}{(Z-1) !}\left(Z_{0}-1\right)^{Z-1} e^{-\left(Z_{0}-1\right)} .
$$


The variance of $Z$ is related to the average of $Z$ as

$$
\left\langle\left(Z-Z_{0}\right)^{2}\right\rangle_{\mathrm{eq}}=Z_{0}-1 \approx Z_{0} \quad\left(\text { for } Z_{0} \gg 1\right)
$$

The segment number distribution function is expressed as

$$
\begin{aligned}
P_{\mathrm{eq}}(n)= & \sum_{Z=1}^{\infty} \int d\left\{\boldsymbol{R}_{k}\right\} d\left\{N_{k}\right\}\left[\frac{1}{Z} \sum_{l=1}^{Z} \delta\left(n-N_{l}\right)\right] \\
& \times P_{\mathrm{eq}}\left(\left\{\boldsymbol{R}_{k}\right\},\left\{N_{k}\right\}, Z\right) \\
= & e^{-\xi_{0}} \sum_{Z=1}^{\infty} \frac{\xi_{0}^{Z-1}}{Z N} \sum_{l=1}^{Z} \frac{1}{N^{Z-2}} \int d\left\{N_{k}\right\} \\
& \times \delta\left(n-N_{l}\right) \delta\left(N-\sum_{k=1}^{Z} N_{k}\right)
\end{aligned}
$$

The integrals over $\left\{N_{k}\right\}$ can be calculated to be

$$
\begin{array}{r}
\frac{1}{N^{Z-2}} \int d\left\{N_{k}\right\} \delta\left(n-N_{l}\right) \delta\left(N-\sum_{k=1}^{Z} N_{k}\right) \\
= \begin{cases}N \delta(n-N) & (Z=1), \\
\frac{1}{(Z-2) !}\left(1-\frac{n}{N}\right)^{Z-2} & (Z \geq 2) .\end{cases}
\end{array}
$$

Thus, Eq. (A9) can be reduced to the following form:

$$
\begin{aligned}
P_{\mathrm{eq}}(n) & =e^{-\left(Z_{0}-1\right)} \delta(n-N)+\frac{Z_{0}-1}{N} \exp \left[-\left(Z_{0}-1\right) \frac{n}{N}\right] \\
& =e^{1-N / N_{0}} \delta(n-N)+\frac{N-N_{0}}{N_{0} N} e^{-n\left(N-N_{0}\right) / N N_{0}}
\end{aligned}
$$

where we used $Z_{0}=N / N_{0}$ (notice that, this $N_{0}$ is different from $N_{e}$ in Ref. 24). For large $Z_{0}$, Eq. (A11) can be approximated by the exponential function

$$
P_{\mathrm{eq}}(n) \approx \frac{1}{N_{0}} e^{-n / N_{0}}
$$

The bond vector distribution function can be calculated in a similar way,

$$
\begin{aligned}
P_{\mathrm{eq}}(\boldsymbol{Q})= & \sum_{Z=1}^{\infty} \int d\left\{\boldsymbol{R}_{k}\right\} d\left\{N_{k}\right\}\left[\frac{1}{Z} \sum_{l=1}^{Z} \delta\left(\boldsymbol{Q}-\boldsymbol{R}_{l}+\boldsymbol{R}_{l-1}\right)\right] \\
& \times P_{\mathrm{eq}}\left(\left\{\boldsymbol{R}_{k}\right\},\left\{N_{k}\right\}, Z\right) \\
= & \left(\frac{3}{2 \pi b^{2}}\right)^{3 / 2} \int_{0}^{N} d n \frac{1}{n^{3 / 2}} e^{-3 Q^{2} / 2 n b^{2}} P_{\mathrm{eq}}(n) .
\end{aligned}
$$

Substituting Eq. (A11) into Eq. (A13), we have

$$
\begin{aligned}
P_{\mathrm{eq}}(\boldsymbol{Q})= & \left(\frac{3}{2 \pi N b^{2}}\right)^{3 / 2} e^{-3 \boldsymbol{Q}^{2} / 2 N b^{2}-N / N_{0}+1} \\
& +\left(\frac{3}{2 \pi N_{0} b^{2}}\right)^{3 / 2} \frac{N-N_{0}}{N_{0} N} \int_{0}^{N} d n\left(\frac{N_{0}}{n}\right)^{3 / 2} \\
& \times \exp \left[-\frac{3 \boldsymbol{Q}^{2}}{2 n b^{2}}-\frac{N-N_{0}}{N_{0} N} n\right] \\
= & \left(\frac{3}{2 \pi N b^{2}}\right)^{3 / 2} e^{-3 \boldsymbol{Q}^{2} / 2 N b^{2}-N / N_{0}+1} \\
& +\frac{3}{4 \pi b^{2}} \frac{N-N_{0}}{N_{0} N} \frac{1}{|\boldsymbol{Q}|}\left[\exp \left(\sqrt{\frac{6}{b^{2}}} \frac{N-N_{0}}{N_{0} N}|\boldsymbol{Q}|\right)\right. \\
& \times \operatorname{erfc}\left(\sqrt{\frac{3}{2 N b^{2}}}|\boldsymbol{Q}|+\sqrt{\left.\frac{N}{N_{0}}-1\right)}\right. \\
& +\exp \left(-\sqrt{\frac{6}{b^{2}}} \frac{N-N_{0}}{N_{0} N}|\boldsymbol{Q}|\right) \\
& \times \operatorname{erfc}\left(\sqrt{\frac{3}{2 N b^{2}}}|\boldsymbol{Q}|-\sqrt{\left.\frac{N}{N_{0}}-1\right)}\right),
\end{aligned}
$$

where erfc $x$ is the complementary error function. ${ }^{25}$ (See Appendix B 2 for details.) Although Eq. (A14) is exact, it is quite complicated and not intuitive. It can be reduced to the following simple approximate form for sufficiently large $Z_{0}$ :

$$
P_{\mathrm{eq}}(\boldsymbol{Q}) \approx \frac{3}{2 \pi N_{0} b^{2}|\boldsymbol{Q}|} \exp \left(-\sqrt{\frac{6}{N_{0} b^{2}}}|\boldsymbol{Q}|\right) .
$$

The bond length distribution function can be expressed as

$$
P_{\mathrm{eq}}(Q) \approx \frac{6 Q}{N_{0} b^{2}} \exp \left(-\sqrt{\frac{6}{N_{0} b^{2}}} Q\right) .
$$

Equation (A16) has a maximum at $Q / \sqrt{N_{0} b^{2}}=1 / \sqrt{6}$.

As shown in the main text, the distribution functions for the single chain non-interacting slip-link model are qualitatively different from the ones for the single chain repulsive slip-link model. Such differences arise only from the difference of the interaction between neighboring slip-links.

\section{APPENDIX B: CALCULATIONS OF INTEGRALS}

In this appendix, we show the detailed calculations for several integrals appear during the derivation of distribution functions in the main text. Although the calculations are complicated, we can obtain the analytic expressions by using special functions such as the gamma function or the modified Bessel function.

\section{Integrals over $\left\{N_{k}\right\}$}

First, we show the detailed calculation of the integral, which appears in Eq. (38),

$$
I_{1} \equiv \frac{1}{N^{5 Z / 2-1}} \int d\left\{N_{k}\right\} \delta\left(N-\sum_{k=1}^{Z} N_{k}\right) \prod_{k=1}^{Z} N_{k}^{3 / 2}
$$


To calculate the integral over $\left\{N_{k}\right\}$, we introduce the following variable transform:

$$
s_{k}= \begin{cases}0 & (k=0), \\ \left(N_{k}-N_{k-1}\right) / N & (k=1,2, \ldots, Z-1), \\ 1 & (k=Z) .\end{cases}
$$

The integral over $\left\{N_{k}\right\}$ with the delta function can be transformed into an integral over $\left\{s_{k}\right\}$ without a delta function,

$$
\frac{1}{N^{Z-1}} \prod_{k=1}^{Z} \int_{0}^{N} d N_{k} \delta\left(N-\sum_{k=1}^{Z} N_{k}\right)=\prod_{k=1}^{Z-1} \int_{0}^{s_{k+1}} d s_{k} .
$$

Equation (B1) can be modified as follows, by using the transform (B3):

$$
I_{1}=\prod_{k=1}^{Z-1} \int_{0}^{s_{k+1}} d s_{k} \prod_{l=0}^{Z-1}\left(s_{l+1}-s_{l}\right)^{3 / 2}
$$

We consider the integral over $s_{1}$ in Eq. (B4). It can be modified as

$$
\int_{0}^{s_{2}} d s_{1}\left(s_{2}-s_{1}\right)^{3 / 2} s_{1}^{3 / 2}=B(5 / 2,5 / 2) s_{2}^{4},
$$

where $B(x, y)$ is the beta function. ${ }^{25}$ Similarly, the integral over $s_{2}$ in Eq. (B4) can be calculated to be

$$
\begin{gathered}
\int_{0}^{s_{3}} d s_{2}\left(s_{3}-s_{2}\right)^{3 / 2}\left[B(5 / 2,5 / 2) s_{2}^{4}\right] \\
=B(5 / 2,5 / 2) B(5 / 2,10 / 2) s_{3}^{13 / 2} .
\end{gathered}
$$

Iterating the same procedure, finally we have the following expression for $I_{1}$ :

$$
I_{1}=\prod_{k=1}^{Z-1} B(5 / 2,5 k / 2)=\prod_{k=1}^{Z-1} \frac{\Gamma(5 / 2) \Gamma(5 k / 2)}{\Gamma(5(k+1) / 2)}=\frac{\Gamma^{Z}(5 / 2)}{\Gamma(5 Z / 2)},
$$

where we have utilized the relation between the beta and gamma functions, ${ }^{25}$

$$
B(x, y)=\frac{\Gamma(x) \Gamma(y)}{\Gamma(x+y)} .
$$

By substituting $\Gamma(5 / 2)=3 \sqrt{\pi} / 4$ into Eq. (B7), we have Eq. (38).

Next, we show the detailed calculation of the integral in Eq. (48),

$$
\begin{aligned}
I_{2} \equiv & \frac{1}{N^{5 Z / 2-2}} \int d\left\{N_{k}\right\}\left[\frac{1}{Z} \sum_{l=1}^{Z} \delta\left(n-N_{l}\right)\right] \\
& \times \delta\left(N-\sum_{k=1}^{Z} N_{k}\right) \prod_{k=1}^{Z} N_{k}^{3 / 2} \\
= & \frac{1}{Z} \sum_{l=1}^{Z} \prod_{k=1}^{Z-1} \int_{0}^{s_{k+1}} d s_{k} \delta\left(\tilde{n}-\left(s_{l+1}-s_{l}\right)\right) \prod_{m=0}^{Z-1}\left(s_{m+1}-s_{m}\right)^{3 / 2},
\end{aligned}
$$

where we defined $\tilde{n} \equiv n / N$. The integral over $\left\{s_{k}\right\}$ can be performed in the similar way to the case of $I_{1}$,

$$
\begin{aligned}
I_{2}= & \frac{1}{Z} \sum_{l=1}^{Z} \prod_{k=l+1}^{Z-1} \int_{0}^{s_{k+1}} d s_{k} \prod_{m=l+1}^{Z-1}\left(s_{m+1}-s_{m}\right)^{3 / 2} \\
& \times \int_{0}^{s_{l+1}} d s_{l} \delta\left(\tilde{n}-\left(s_{l+1}-s_{l}\right)\right)\left(s_{l+1}-s_{l}\right)^{3 / 2} s_{l}^{(5 l-2) / 2} \\
& \times \prod_{m=1}^{l-1} B(5 m / 2,5 / 2)=\frac{\tilde{n}^{3 / 2}}{Z} \sum_{l=1}^{Z} \prod_{k=l+1}^{Z-1} \int_{\tilde{n}}^{s_{k+1}} d s_{k} \\
& \times \prod_{m=l+1}^{Z-1}\left(s_{m+1}-s_{m}\right)^{3 / 2}\left(s_{l+1}-\tilde{n}\right)^{(5 l-2) / 2} \prod_{m=1}^{l-1} B(5 m / 2,5 / 2) .
\end{aligned}
$$

To simplify the expression, we introduce another variable transform, $u_{k}=s_{k}-\tilde{n}$. Then, we have

$$
\begin{aligned}
I_{2}= & \frac{\tilde{n}^{3 / 2}}{Z} \sum_{l=1}^{Z} \prod_{k=l+1}^{Z-1} \int_{0}^{\tilde{u}_{k+1}} d u_{k} \prod_{m=l+1}^{Z-1}\left(u_{m+1}-u_{m}\right)^{3 / 2} u_{l+1}^{(5 l-2) / 2} \\
& \times \prod_{m=1}^{l-1} B(5 m / 2,5 / 2) \\
= & \frac{\tilde{n}^{3 / 2}}{Z} \sum_{l=1}^{Z}(1-\tilde{n})^{[5(Z-1)-2] / 2} \prod_{m=1}^{Z-2} B(5 m / 2,5 / 2) . \quad(\mathrm{B} 11)
\end{aligned}
$$

Finally, we have the following expression for $I_{2}$ :

$$
I_{2}=\tilde{n}^{3 / 2}(1-\tilde{n})^{5(Z-1) / 2-1} \frac{\Gamma^{Z-1}(5 / 2)}{\Gamma(5(Z-1) / 2)} .
$$

Equation (B12) together with $\tilde{n}=n / N$ and $\Gamma(5 / 3)=3 \sqrt{\pi} / 4$ gives Eq. (48).

\section{Integrals over $n$}

Here, we show the detailed calculation of Eq. (56). The integral over $n$ in Eq. (56) can be modified as follows, by using the variable transform $t=\sqrt{5 b^{2} / 3 N_{0} Q^{2}} n$ :

$$
\begin{aligned}
I_{3} & \equiv \frac{1}{N_{0}} \int_{0}^{\infty} d n \exp \left(-\frac{3 Q^{2}}{2 n b^{2}}-\frac{5}{2} \frac{n}{N_{0}}\right) \\
& =\sqrt{\frac{3 Q^{2}}{5 N_{0} b^{2}}} \int_{0}^{\infty} d t \exp \left[-\frac{1}{2} \sqrt{\frac{15 Q^{2}}{N_{0} b^{2}}}\left(\frac{1}{t}+t\right)\right] .
\end{aligned}
$$

The integral in Eq. (B13) can be reduced to the first-order modified Bessel function of the second kind. The integral expression of the first-order modified Bessel function of the second kind becomes ${ }^{25}$

$$
\begin{aligned}
K_{1}(x) & =\int_{0}^{\infty} d u e^{-x \cosh u} \cosh u=\frac{1}{2} \int_{-\infty}^{\infty} d u e^{u-x \cosh u} \\
& =\frac{1}{2} \int_{0}^{\infty} d s \exp \left[-\frac{x}{2}\left(s+\frac{1}{s}\right)\right],
\end{aligned}
$$

where we have used the variable transform $s=e^{-u}$. By substituting $x=\sqrt{15 Q^{2} / N_{0} b^{2}}$ into Eq. (B14), we have the 
following expression for $I_{3}$ :

$$
I_{3}=\frac{2}{5} \sqrt{\frac{15 Q^{2}}{N_{0} b^{2}}} K_{1}\left(\sqrt{\frac{15 Q^{2}}{N_{0} b^{2}}}\right) .
$$

This gives Eq. (56).

A similar integral also appears in Eq. (A14). The integral in Eq. (A14) can be calculated as follows:

$$
\begin{aligned}
I_{4} & \equiv \frac{N-N_{0}}{N_{0} N} \int_{0}^{N} d n\left(\frac{N_{0}}{n}\right)^{3 / 2} \exp \left[-\frac{3 Q^{2}}{2 n b^{2}}-\frac{N-N_{0}}{N_{0} N} n\right] \\
& =\frac{2\left(Z_{0}-1\right)}{Z_{0}^{3 / 2}} \int_{1}^{\infty} d t \exp \left[-\frac{3 Q^{2}}{2 N b^{2}} t^{2}-\left(Z_{0}-1\right) \frac{1}{t^{2}}\right],
\end{aligned}
$$

where we used the variable transform $t=\sqrt{N / n}$. The integral over $t$ in Eq. (B16) can be calculated by using the following formula: ${ }^{25}$

$$
\begin{aligned}
\int_{1}^{\infty} d t e^{-x t^{2}-y / t^{2}}= & \frac{\sqrt{\pi}}{4 \sqrt{x}}\left[e^{2 \sqrt{x y}} \operatorname{erfc}(\sqrt{x}+\sqrt{y})\right. \\
& \left.+e^{-2 \sqrt{x y}} \operatorname{erfc}(\sqrt{x}-\sqrt{y})\right]
\end{aligned}
$$

where erfc $x$ is the complementary error function. ${ }^{25}$ Finally, Eq. (B16) can be reduced to

$$
\begin{aligned}
I_{4}= & \frac{\sqrt{\pi}\left(Z_{0}-1\right)}{Z_{0}^{3 / 2}} \sqrt{\frac{N b^{2}}{6 Q^{2}}}\left[\operatorname { e x p } \left[\sqrt{\left.\frac{6 Q^{2}}{N b^{2}}\left(Z_{0}-1\right)\right]}\right.\right. \\
& \times \operatorname{erfc}\left(\sqrt{\frac{3 Q^{2}}{2 N b^{2}}}+\sqrt{Z_{0}-1}\right)+\exp \left[-\sqrt{\frac{6 Q^{2}}{N b^{2}}\left(Z_{0}-1\right)}\right] \\
& \left.\times \operatorname{erfc}\left(\sqrt{\frac{3 Q^{2}}{2 N b^{2}}}-\sqrt{Z_{0}-1}\right)\right] .
\end{aligned}
$$

This gives Eq. (A14).

\section{APPENDIX C: CALCULATIONS OF SADDLE POINT APPROXIMATIONS}

\section{Saddle point approximation for $P_{\mathrm{eq}}(Z)$}

A simple approximate expression is demanding to analyze the statistical properties of the repulsive slip-link model. Here, we attempt to calculate the approximate expression for Eq. (43), for sufficiently large $Z_{0}$. For convenience, we approximate $P_{\mathrm{eq}}(Z)$ by a continuum distribution. The summation over $Z$ is replaced by the integral as follows:

$$
\sum_{Z=1}^{\infty} \approx \int_{-\infty}^{\infty} d Z
$$

We can utilize Stirling's formula ${ }^{25}$ to approximate the gamma function,

$$
\begin{aligned}
\frac{\xi^{Z-1}}{\Gamma(5 Z / 2)} \approx & \exp \left[(Z-1) \ln \xi-\left(\frac{5 Z}{2}-\frac{1}{2}\right) \ln \frac{5 Z}{2}\right. \\
& \left.+\frac{5 Z}{2}-\frac{1}{2} \ln (2 \pi)\right] .
\end{aligned}
$$

Next, we expand the exponent around the saddle point,

$$
\begin{aligned}
f(Z) & \equiv(Z-1) \ln \xi-\left(\frac{5 Z}{2}-\frac{1}{2}\right) \ln \frac{5 Z}{2}+\frac{5 Z}{2}-\frac{1}{2} \ln (2 \pi) \\
& \approx f\left(Z^{*}\right)+\frac{1}{2} f^{\prime \prime}\left(Z^{*}\right)\left(Z-Z^{*}\right)^{2},
\end{aligned}
$$

where $Z^{*}$ is the saddle point value of $Z$, which satisfies the saddle point equation,

$$
f^{\prime}\left(Z^{*}\right)=\ln \xi-\frac{5}{2} \ln \frac{5 Z^{*}}{2}+\frac{1}{2 Z^{*}}=0 .
$$

The distribution function (Eq. (43)) can be approximated as a Gaussian

$$
\begin{aligned}
P_{\mathrm{eq}}(Z) & \approx \frac{\exp \left[f^{\prime \prime}\left(Z^{*}\right)\left(Z-Z^{*}\right)^{2} / 2\right]}{\int_{-\infty}^{\infty} d Z \exp \left[f^{\prime \prime}\left(Z^{*}\right)\left(Z-Z^{*}\right)^{2} / 2\right]} \\
& =\sqrt{\frac{5 Z^{*}+1}{4 \pi\left(Z^{*}\right)^{2}}} \exp \left[-\frac{5 Z^{*}+1}{4\left(Z^{*}\right)^{2}}\left(Z-Z^{*}\right)^{2}\right] .
\end{aligned}
$$

The average value of $Z$ is calculated to be

$$
\langle Z\rangle_{\mathrm{eq}}=Z_{0} \approx Z^{*} \text {. }
$$

Therefore, we can replace $Z^{*}$ in Eq. (C5) by $Z_{0}$. Finally we have Eq. (44) as the approximate form for the subchain number distribution function.

\section{Saddle point approximation for $\boldsymbol{P}_{\mathrm{eq}}(\boldsymbol{n})$}

We consider to obtain a simple approximate expression for Eq. (50). For sufficiently large $Z_{0}$, we can use the following approximate form:

$$
\begin{aligned}
& \sum_{Z=2}^{\infty}\left(1-\frac{n}{N}\right)^{5(Z-1) / 2} \frac{\xi^{Z-1}}{\Gamma(5(Z-1) / 2)} \\
& \quad \approx \int_{-\infty}^{\infty} d Z \exp \left[g\left(Z^{* *}\right)+\frac{1}{2} g^{\prime \prime}\left(Z^{* *}\right)\left(Z-Z^{* *}\right)^{2}\right] \\
& \quad=\sqrt{\frac{2 \pi}{-g^{\prime \prime}\left(Z^{* *}\right)}} e^{g\left(Z^{* *}\right)}
\end{aligned}
$$

where we defined

$$
\begin{aligned}
g(Z) \equiv & \frac{5 Z}{2} \ln \left(1-\frac{n}{N}\right)+Z \ln \xi-\frac{5 Z-1}{2} \ln \frac{5 Z}{2}+\frac{5 Z}{2} \\
& -\frac{1}{2} \ln (2 \pi)
\end{aligned}
$$

and $Z^{* *}$ is given via the following saddle point equation:

$$
g^{\prime}\left(Z^{* *}\right)=\frac{5}{2} \ln \left(1-\frac{n}{N}\right)+\ln \xi-\frac{5}{2} \ln \frac{5 Z^{* *}}{2}+\frac{1}{2 Z^{* *}}=0 .
$$

From Eqs. (C4) and (C6), we have the following approximate relation between $Z^{* *}$ and $Z_{0}$ :

$$
Z^{* *} \approx\left(1-\frac{n}{N}\right) Z_{0}
$$


Then, we can write

$$
\begin{aligned}
& \sum_{Z=2}^{\infty}\left(1-\frac{n}{N}\right)^{5(Z-1) / 2} \frac{\xi^{Z-1}}{\Gamma(5(Z-1) / 2)} \approx \frac{Z_{0}}{\sqrt{e}}\left(1-\frac{n}{N}\right) \\
& \quad \times \exp \left[\frac{5 Z_{0}}{2}\left(1-\frac{n}{N}\right)\right],
\end{aligned}
$$

and the segment number distribution function can be expressed approximately as

$$
\begin{aligned}
P_{\text {eq }}(n) \propto & \left(\frac{n}{N}\right)^{3 / 2}\left(1-\frac{n}{N}\right)^{-1}\left[\left(1-\frac{n}{N}\right)\right. \\
& \left.\times \exp \left[\frac{5 Z_{0}}{2}\left(1-\frac{n}{N}\right)\right]\right] \propto n^{3 / 2} \exp \left(-\frac{5 n}{2 N_{0}}\right) .
\end{aligned}
$$

By normalizing Eq. (C12), finally we have Eq. (51) as the approximate form for $P_{\mathrm{eq}}(n)$.

\section{APPENDIX D: STRONG REPULSION LIMIT}

In the main text and Appendix A, we calculated some equilibrium distribution functions for repulsive and noninteracting slip-link models, respectively. For comparison, here we consider the case where the repulsive interaction between slip-links is quite strong. We call such a limit as the strong repulsion limit. As we will show, slip-links form a Wigner crystal like ordered structure in this limit.

We assume the following effective potential for sliplinks, instead of Eq. (30):

$$
\tilde{U}_{\text {slip-link }}(n)=-\alpha k_{B} T \ln n .
$$

Here, $\alpha$ is the parameter which represents the strength of the repulsive interaction. ( $\alpha=0$ and $\alpha=3 / 2$ correspond to the non-interacting and repulsive slip-link models, respectively.) At the strong repulsion limit, we set $\alpha \rightarrow \infty$.

The equilibrium probability distribution function and the grand partition function are expressed as follows:

$$
\begin{aligned}
& P_{\mathrm{eq}}\left(\left\{\boldsymbol{R}_{k}\right\},\left\{N_{k}\right\}, Z\right)=\frac{e^{\epsilon Z / k_{B} T}}{\Xi \Lambda^{3(Z+1)} N^{Z-1}} \delta\left(N-\sum_{k=1}^{Z} N_{k}\right) \\
& \times \prod_{k=1}^{Z} N_{k}^{\alpha-3 / 2} \exp \left[-\frac{3\left(\boldsymbol{R}_{k}-\boldsymbol{R}_{k-1}\right)^{2}}{2 N_{k} b^{2}}\right], \\
& \Xi \equiv \sum_{Z=1}^{\infty} \frac{e^{\epsilon Z / k_{B} T}}{\Lambda^{3(Z+1)} N^{Z-1}} \int d\left\{\boldsymbol{R}_{k}\right\} d\left\{N_{k}\right\} \delta\left(N-\sum_{k=1}^{Z} N_{k}\right) \\
& \quad \times \prod_{k=1}^{Z} N_{k}^{\alpha-3 / 2} \exp \left[-\frac{3\left(\boldsymbol{R}_{k}-\boldsymbol{R}_{k-1}\right)^{2}}{2 N_{k} b^{2}}\right] .
\end{aligned}
$$

Since the repulsive interaction energy becomes very large at the strong repulsion limit, we can reasonably utilize the saddle point approximation for $\left\{N_{k}\right\}$. For $\alpha \gg 1$, the distribution of $\left\{N_{k}\right\}$ is expected to be sufficiently sharp, and thus, the fluctuations around the saddle point is negligible. Then, the grand partition function can be approximated as

$$
\begin{aligned}
\Xi \approx & \sum_{Z=1}^{\infty} \frac{e^{\epsilon Z / k_{B} T}}{\Lambda^{3(Z+1)} N^{Z-1}} \int d\left\{\boldsymbol{R}_{k}\right\}\left(\frac{N}{Z}\right)^{(\alpha-3 / 2) Z} \\
& \times \exp \left[-\frac{3 Z\left(\boldsymbol{R}_{k}-\boldsymbol{R}_{k-1}\right)^{2}}{2 N b^{2}}\right] \\
= & \frac{\mathcal{V} N}{\Lambda^{3}} \sum_{Z=1}^{\infty}\left[\left(\frac{2 \pi b^{2}}{3 \Lambda^{2}}\right)^{3 / 2} \frac{N^{\alpha-1}}{Z^{\alpha}} e^{\epsilon / k_{B} T}\right]^{Z} .
\end{aligned}
$$

For simplicity, we assume that the average number of subchains is sufficiently large $\left(Z_{0} \gg 1\right)$ and utilize the saddle point approximation for $Z$. The result is

$$
\begin{aligned}
& P_{\mathrm{eq}}(Z) \approx\left(\frac{\alpha}{2 \pi Z_{0}}\right)^{1 / 2} \exp \left[-\frac{\alpha}{2 Z_{0}}\left(Z-Z_{0}\right)^{2}\right] \rightarrow \delta\left(Z-Z_{0}\right) \\
& (\alpha \rightarrow \infty) .
\end{aligned}
$$

Thus, we find that the subchain number distribution simply becomes the delta function. This means that there is essentially no subchain number fluctuation in the strong repulsion limit. Similarly, the segment number distribution function is obtained as

$$
P_{\mathrm{eq}}(n) \approx \delta\left(n-N_{0}\right)
$$

Equation (D6) means that the chemical distance between two neighboring slip-links is constant (slip-links are placed equidistantly on a chain). Equations (D5) and (D6) are naturally derived from the dependence of the variances of $Z$ and $n$ on $\alpha$. From the results in Appendix A, the main text, and this appendix, the variances are roughly estimated as $\left\langle\left(Z-Z_{0}\right)^{2}\right\rangle_{\mathrm{eq}} \approx Z_{0} /(1+\alpha)$ and $\left\langle\left(n-N_{0}\right)^{2}\right\rangle_{\mathrm{eq}} \approx N_{0}^{2} /(1+$ $\alpha)$. At the strong repulsion limit $(\alpha \rightarrow 0)$, both of them approach to zero and the distribution functions reduce to delta functions (as Eqs. (D5) and (D6)). We can interpret such a state as a sort of Wigner crystal. This situation seems to be qualitatively different from most of the slip-link models, such as the PCN model. It would rather be similar to the simple tube model $^{3}$ in which each tube segment contains a constant number of segments. (But, of course, the slip-link model is not equivalent to the tube model.) Anyway, the distribution functions for $Z$ and $n$ at the strong repulsion limit are much sharper than the distribution functions for the non-interacting or repulsive slip-link models. Thus, we consider that these distribution functions become sharper as the repulsive interaction between slip-links increases.

Because the segment number $n$ is fixed to $N_{0}$, the bond vector distribution function is nothing but a Gaussian. The bond vector and bond length distribution functions become

$$
\begin{gathered}
P_{\mathrm{eq}}(\boldsymbol{Q}) \approx\left(\frac{3}{2 \pi N_{0} b^{2}}\right)^{3 / 2} \exp \left(-\frac{3 Q^{2}}{2 N_{0} b^{2}}\right), \\
P_{\mathrm{eq}}(Q) \approx \frac{4}{\sqrt{\pi}}\left(\frac{3}{2 N_{0} b^{2}}\right)^{3 / 2} Q^{2} \exp \left(-\frac{3 Q^{2}}{2 N_{0} b^{2}}\right) .
\end{gathered}
$$

At the limit of small $Q$, we have $P_{\text {eq }}(Q) \propto Q^{2}$ as the asymptotic form. This asymptotic behavior is similar to one of the 
repulsive slip-link model (Eq. (59)). Equation (D8) has a maximum at $Q / \sqrt{N_{0} b^{2}}=\sqrt{2 / 3} \approx 0.816$, which is larger than the value for the repulsive slip-link model. Thus, we expect that the value of $Q / \sqrt{N_{0} b^{2}}$ which gives the maximum increases as the repulsive interaction increases.

${ }^{1}$ Y. Masubuchi, J. Takimoto, K. Koyama, G. Ianniruberto, F. Greco, and G. Marrucci, J. Chem. Phys. 115, 4387 (2001).

${ }^{2}$ Y. Masubuchi, G. Ianniruberto, F. Greco, and G. Marrucci, J. Non-Newtonian Fluid Mech. 149, 87 (2008).

${ }^{3} \mathrm{M}$. Doi and S. F. Edwards, The Theory of Polymer Dynamics (Oxford University Press, Oxford, 1986).

${ }^{4}$ Y. Masubuchi, G. Ianniruberto, F. Greco, and G. Marrucci, Rheol. Acta 46, 297 (2006).

${ }^{5}$ Y. Masubuchi, T. Yaoita, Y. Matsumiya, and H. Watanabe, J. Chem. Phys. 134, 194905 (2011)

${ }^{6}$ Y. Masubuchi, H. Watanabe, G. Ianniruberto, F. Greco, and G. Marrucci, Macromolecules 41, 8275 (2008).

${ }^{7}$ R. Everaers, S. K. Sukumaran, G. S. Grest, C. Svaneborg, A. Sivasubramanian, and K. Kremer, Science 303, 823 (2004).

${ }^{8}$ M. Kröger, Comput. Phys. Commun. 168, 209 (2005).

${ }^{9}$ C. Tzoumanekas and D. N. Theodorou, Macromolecules 39, 4592 (2006).

${ }^{10}$ Y. Masubuchi, T. Uneyama, H. Watanabe, G. Ianniruberto, F. Greco, and G. Marrucci, J. Chem. Phys. 132, 134902 (2010).

${ }^{11}$ R. Kubo, M. Toda, and N. Hashitsume, Statistical Physics II (Springer, Heidelberg, 1991).

${ }^{12}$ D. J. Evans and G. P. Morris, Statistical Mechanics of Nonequilibrium Liquids, 2nd ed. (Cambridge University Press, Cambridge, 2008).
${ }^{13}$ H. Risken, The Fokker-Planck Equation, 2nd ed. (Springer, Berlin, 1989).

${ }^{14}$ D. M. Nair and J. D. Schieber, Macromolecules 39, 3386 (2006).

${ }^{15}$ T. Uneyama, Nihon Reoroji Gakkaishi 39, 135 (2011).

${ }^{16}$ T. Uneyama and K. Horio, J. Polym. Sci., Part B: Polym. Phys. 49, 966 (2011).

${ }^{17}$ T. Yaoita, T. Isaki, Y. Masubuchi, H. Watanabe, G. Ianniruberto, F. Greco, and G. Marrucci, J. Chem. Phys. 128, 154901 (2008).

${ }^{18}$ C. W. Gardiner, Handbook of Stochastic Methods, 3rd ed. (Springer, Berlin, 2004).

${ }^{19}$ K. Kawasaki, J. Phys. A 6, 1289 (1973).

${ }^{20}$ K. Sekimoto, Stochastic Energetics, Lecture Notes in Physics Vol. 799 (Springer, Berlin, 2010).

${ }^{21}$ P. Kindt and W. J. Briels, J. Chem. Phys. 127, 124901 (2007).

${ }^{22}$ A. N. Beris and B. J. Edwards, Thermodynamics of Flowing Systems (Oxford University Press, Oxford, 1994).

${ }^{23}$ H. C. Öttinger, Phys. Rev. E 57, 1416 (1998).

${ }^{24}$ J. D. Schieber, J. Chem. Phys. 118, 5162 (2003).

${ }^{25}$ Handbook of Mathematical Functions with Formulas, Graphs, and Mathematical Tables, 10th ed., edited by M. Abramowitz and I. A. Stegun (Dover, New York, 1972).

${ }^{26}$ E. W. Weisstein, “Mittag-Leffler Function” From MathWorld A Wolfram Web Resource; see http://mathworld.wolfram.com/MittagLefflerFunction.html.

${ }^{27}$ S. Okuda, Y. Inoue, Y. Masubuchi, T. Uneyama, and M. Hojo, "Soft-core interaction between entanglement segments for primitive chain network simulations," Rheol. Acta (submitted).

${ }^{28}$ D. S. Dean, J. Phys. A 29, L613 (1996).

${ }^{29}$ T. Uneyama, J. Chem. Phys. 126, 114902 (2007).

${ }^{30}$ F. Greco, Eur. Phys. J. E 25, 175 (2008).

${ }^{31}$ M. Doi and J. Takimoto, Philos. Trans. R. Soc. London, Ser. A 361, 641 (2003). 